Canadian Journal of Physics

Canadian

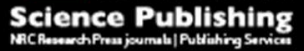

Revue canadienne de physique

\title{
Electric Dipole Transitions between Low-Lying Levels in Doubly Ionized Krypton, Xenon, and Radon
}

\begin{tabular}{|r|l|}
\hline Journal: & Canadian Journal of Physics \\
\hline Manuscript ID & cjp-2017-0238.R1 \\
\hline Manuscript Type: & Article \\
\hline Date Submitted by the Author: & 22-Jun-2017 \\
\hline Complete List of Authors: & $\begin{array}{l}\text { Eser, Selda; Sakarya University, Physics } \\
\text { Özdemir, Leyla; Sakarya University, Physics }\end{array}$ \\
\hline Keyword: & $\begin{array}{l}\text { Energies, correlation effects, Breit interactions, QED contributions, E1 } \\
\text { transitions }\end{array}$ \\
\hline $\begin{array}{r}\text { Is the invited manuscript for } \\
\text { consideration in a Special } \\
\text { Issue? : }\end{array}$ & N/A \\
\hline
\end{tabular}




\title{
Electric Dipole Transitions between Low-Lying Levels in Doubly Ionized Krypton, Xenon, and Radon
}

\author{
Selda Eser $^{1}$ and Leyla Özdemir \\ Department of Physics, Sakarya University, 54187, Sakarya, Turkey
}

\begin{abstract}
Using the general-purpose relativistic atomic structure package (GRASP) based on a fully relativistic multiconfiguration Dirac-Fock (MCDF) method, the transition parameters such as transition rates (probabilities), oscillator strengths, and line strengths for the electric dipole (E1) transitions between low-lying levels are evaluated for doubly ionized krypton, xenon, and radon. Breit interactions for relativistic effects and quantum electrodynamical (QED) contributions besides valence and valence-core correlation effects are taken into account in calculations. We compare the results obtained with the available data in literature and discuss them, when possible.
\end{abstract}

Key words: Energies, correlation effects, Breit interactions, QED contributions, E1 transitions

PACS number: 31.15.ag; 31.15.V; 31.15.aj; 31. 30.-i; 32.70.Cs

\footnotetext{
${ }^{1}$ Corresponding author.

E-mail: $\underline{\text { skabakci@sakarya.edu.tr }}$ (S. Eser)
} 


\section{Introduction}

Radiative transition parameters, such as transition probabilities, oscillator strengths and line strengths are fundamental characteristics of excited states of atoms and ions. These data plays an important role in plasma and laser investigations; and astrophysics. To interpret observed spectra, knowledge of accurate transition parameters is necessary [1].

There are a few electric dipole transition data for $\mathrm{Kr}$ III and $\mathrm{Xe}$ III; even no data for $\mathrm{Rn}$ III in literature. Krypton $(Z=36)$, xenon $(Z=54)$ and radon $(Z=86)$ atoms are among the noble gases. The doubly ionized krypton (Kr III), xenon (Xe III) and radon (Rn III) atoms are isoelectronic with neutral selenium, tellurium and polonium, respectively. These ions have $n \mathrm{~s}^{2} n \mathrm{p}^{4}$ electron ground configuration ( $n=4,5$, and 6 , for $\mathrm{Kr}$ III, Xe III, and Rn III, respectively). The ground level for ions is $n p^{4}{ }^{3} \mathrm{P}_{2}$, and this level is followed by ${ }^{3} \mathrm{P}_{1},{ }^{3} \mathrm{P}_{0},{ }^{1} \mathrm{D}_{2}$ and ${ }^{1} \mathrm{~S}_{0}$ in the same configuration.

Krypton has been detected in the spectra of the interstellar medium and present in many light sources and lasers as the working gas. In addition, the singly and doubly ionized krypton spectral lines are very useful for plasma diagnostical purposes [2]. There are studies, in particular on radiative lifetimes for metastable states and transition parameters for krypton ions [2-16]. Raineri et al. also reported the weighted oscillator strengths and spectral lines belonging to some transitions and compared multiconfigurational Hartree-Fock relativistic approach [17]. And, Saloman has compiled observed spectral lines of the krypton atom (Kr I-Kr XXXVI) [18].

In the development of lasers and laser techniques, xenon has always had an important role and is also an important element for light sources and development of lamps because of its rich emission spectrum, and Xe III (Te-like) is interesting for astrophysics as well, for example it was identified in the planetary nebula NGC 7027 [19]. For Xe III, there are some studies including lifetimes and radiative transitions parameters, in particular metastable states [14, 15, 20-28]. Then Saloman has compiled the energy levels and observed spectral lines of the xenon atom, in all stages of ionization for which experimental data were available [29]. 
Radon is a radioactive noble gas element, which is obtained by radioactive disintegration of radium, while all other noble gases are present in atmosphere. Biémont and Quinet presented a theoretical study for Rn III [30]. Pernpointner and co-workers reported double ionization spectra of the noble gas atoms Ne through $\mathrm{Rn}[31]$.

In this work, we report radiative transition parameters such as transition rates (probabilities), oscillator strengths, and line strengths for the electric dipole transitions in doubly ionized krypton (Se-like), xenon (Te-like), and radon (Po-like), using the general-purpose relativistic atomic structure package (GRASP) [32]. This code includes Breit interactions (magnetic interaction between the electrons and retardation effects of the electron-electron interaction) for relativistic effects and quantum electrodynamical (QED) contributions (self-energy and vacuum polarization). These contributions are important in investigations include electronic structure and spectroscopic properties of many electron systems. In addition, we have taken into account the configurations including the excitations from valence and core

\section{Computational details}

The general-purpose relativistic atomic structure package, GRASP code [32] is based on a fully relativistic multiconfiguration Dirac-Fock (MCDF), and uses Thomas-Fermi and Coulomb potential for calculate wavefunctions according to JJ and LS coupling. In the MCDF method [33] an atomic state can be expanded as a linear combination of configuration state functions (CSFs)

$$
\Psi_{a}(P J M)=\sum_{r=1}^{n_{c}} C_{r}(\alpha)\left|\gamma_{r}(P J M)\right\rangle
$$

where $n_{c}$ is the number of CSFs included in the evaluation of atomic state functions and $C_{r}$ is the mixing coefficient; and is optimized usually on the basis of the many-electron Dirac-Coulomb Hamiltonian. This method is basic and requires no knowledge of the internal coupling of the CSFs with a given parity $P$ and angular momentum $(J, M)$. The CSFs are the sum of products of single-electron Dirac spinors,

$$
\phi(r, \theta, \varphi, \sigma)=\frac{1}{r}\left(\begin{array}{l}
P(r) \chi_{\kappa m}(\theta, \varphi, \sigma) \\
i Q(r) \chi_{-\kappa m}(\theta, \varphi, \sigma)
\end{array}\right),
$$


where $\kappa$ is a quantum number and $\chi_{\kappa m}$ is the spinor spherical harmonic in the LSJ coupling scheme. The $P(r)$ and $Q(r)$ are large and small radial components of one-electron wavefunctions represented on a logarithmic grid. The energy functional is based on the Dirac-Coulomb Hamiltonian in form

$$
\left.H_{D C}=\sum_{j=1}^{N}\left(C \overrightarrow{\alpha_{j}} \cdot \vec{p}_{j}\right)+\left(\beta_{j}-1\right) c^{2}+V\left(r_{j}\right)\right)+\sum_{j<k}^{N} \frac{1}{r_{j k}} \quad,
$$

where $V(r j)$ is the monopole part of the electron-nucleon interaction. Once initial and final state functions have been calculated, the radiative matrix element for radiative properties computation can be obtained from

$$
O_{i f}=\left\langle\psi(i)\left|\boldsymbol{O}_{q}^{\pi(k)}\right| \psi(f)\right\rangle
$$

where $\boldsymbol{O}_{q}^{\pi(k)}$ is a spherical operator of rank $k$ and parity $\pi$, and $\pi(k)$ is $\pi=(-1)^{k}$, for an electric multipole transition or $\pi=(-1)^{k+1}$, for a magnetic multipole transition. The largest transition probability is for electric dipole (E1) radiation, dominated by the least factor $1 / \alpha^{2}$ over other types of transitions (E2, M1, M2, etc.).

The transition probabilities for the emission from the upper level to the lower level is given by

$$
A^{\pi k}\left(\gamma^{\prime} J^{\prime}, \gamma J\right)=2 C_{k}\left[\alpha\left(E_{\gamma^{\prime} J^{\prime}}-E_{\gamma J}\right)\right]^{2 k+1} \frac{S^{\pi k}\left(\gamma^{\prime} J^{\prime}, \gamma J\right)}{g_{J^{\prime}}}
$$

where $\mathrm{S}^{\pi \mathrm{k}}$ is line strength,

$$
S^{\pi k}\left(\gamma^{\prime} J^{\prime}, \gamma J\right)=\left|<\gamma J\left\|\boldsymbol{O}^{\pi(k)}\right\| \gamma^{\prime} J^{\prime}>\right|^{2}
$$


$C_{k}=(2 k+1)(k+1) / k((2 k+1) ! !)^{2}$, and $\boldsymbol{O}^{\pi(k)}$ is transition operator.

The oscillator strength is dimensionless parameter. It is associated with radiation-induced electric dipole transitions between two states,

$$
f^{\pi k}\left(\gamma J, \gamma^{\prime} J^{\prime}\right)=\frac{1}{\alpha} C_{k}\left[\alpha\left(E_{\gamma^{\prime} J^{\prime}}-E_{\gamma J}\right)\right]^{2 k-1} \frac{S^{\pi k}\left(\gamma J, \gamma^{\prime} J^{\prime}\right)}{g_{J}} .
$$

A similar expression can be written for the emission oscillator strength where $\gamma^{\prime} J^{\prime}$ and $\gamma J$ are interchanged, making the emission oscillator strength negative.

In calculations we have used the option extended average level (EAL) averaging of the expression energy. It is extended to configuration states with not only different total angular momentum but also with different parity. Also, Breit corrections (magnetic interaction between the electrons and retardation effects of the electron-electron interaction), and QED (self-energy and vacuum polarization), and various correlation contributions have been considered. Due to the Coulomb interaction between the electrons, the electron correlation effects are also important, in particular, on fine structure and transitions. Therefore, the configurations including excitations from valence and core have been taken into account (Table 1). QED contributions are self-energy and vacuum polarization, which are also included in the computations of the transition energy. The finite-nucleus effect is taken into account by assuming an extended Fermi distribution for the nucleus. Both the Breit and QED contributions are treated as perturbation and are not included directly in the SCF procedure. The mixing coefficients are calculated by diagonalizing the modified Hamiltonian.

Table 1. Configurations considered for calculations.

\begin{tabular}{l|l}
\hline \hline Ion & Configurations \\
\hline \hline Kr III & $4 s^{2} 4 p^{4}, 4 s 4 p^{5}, 4 p^{6}, 4 s^{2} 4 p^{3} 5 s, 4 p^{5} 5 s, 4 s^{2} 4 p^{3} 6 s, 4 s^{2} 4 p^{3} 5 p$ \\
\hline \multirow{2}{*}{ Xe III } & $\begin{array}{l}5 s^{2} 5 p^{4}, 5 s 5 p^{5}, 5 p^{6}, 5 s 5 p^{4} 5 d, 5 s^{2} 5 p^{2} 5 d^{2}, 5 p^{4} 5 d^{2}, 5 s^{2} 5 p^{3} 5 d, 5 p^{5} 5 d, 5 s^{2} 5 p^{3} 6 s, 5 s^{2} 5 p^{3} 6 p \\
\end{array}$ \\
$5 s^{2} 5 p^{3} 6 d, 5 s^{2} 5 p^{3} 4 f$ \\
\hline \multirow{2}{*}{ Rn III } & $6 s^{2} 6 p^{4}, 6 s 6 p^{5}, 6 p^{6}, 6 s^{2} 6 p^{3} 7 s, 6 s^{2} 6 p^{3} 7 p, 6 s^{2} 6 p^{3} 6 d, 6 p^{4} 6 d^{2}, 6 p^{5} 6 d, 6 s 6 p^{3} 7 s^{2}, 6 s 6 p^{3} 8 s^{2}$, \\
& $6 s^{2} 6 p^{2} 6 d 7 s, 6 p^{4} 7 s^{2}, 6 s^{2} 6 p^{2} 7 s^{2}, 6 p^{5} 7 s, 6 s^{2} 6 p^{3} 7 d, 6 p^{4} 6 d 7 s$ \\
\hline \hline
\end{tabular}




\section{Results and discussion}

Using GRASP code [32], the calculations of transition parameters such as oscillator strengths, $\mathrm{F}_{\mathrm{ji}}$, transition rates (or probabilities), $\mathrm{A}_{\mathrm{ij}}$ (in $\mathrm{s}^{-1}$ ), and line strengths, $\mathrm{S}_{\mathrm{ij}}$ (in a. u.) have been obtained for electric dipole (E1) transitions among levels from configurations selected in Table 1. We have only presented transition parameters for electric dipole (E1) transitions from low-lying levels to ground-state levels in Table 2, Table 3 and Table 4 for Kr III, Xe III and Rn III, respectively. Only odd-parity states are indicated by the superscript " " " in tables. Also, the number in brackets represents the power of 10. Electric dipole transitions are calculated in two gauges. The Babushkin gauge can be shown to reduce non-relativistically to the length form of the matrix element while the Coulomb gauge reduces to the velocity form. We have here presented the results according to the length form. The ratio of velocity and length forms for oscillator strengths have been given in tables. This ratio must be about 1 for accuracy. We have obtained 62, 454, and 393 energy levels, and 670, 19271, and 18983 electric dipole transitions between these levels performed for $\mathrm{Kr}$ III, Xe III, and Rn III, respectively. In Table 2, we have presented transition probabilities, logarithmic of weighted oscillator strengths, line strengths and the ratio of velocity and length forms; and compared the logarithmic oscillator strengths with results reported using MCHF approach by Raineri et al. [17]. As seen from the table, there is good agreement between results, in general. In Table 3 and Table 4, we have given same parameters as in Table 2 except the oscillator strength, $\mathrm{F}_{\mathrm{ji}}$, besides logarithmic weighted oscillator strength, $\log \left(\mathrm{F}_{\mathrm{ji}}\right)$, values. There are no values on electric dipole transition parameter values for the lines presented in Table 3 and Table 4 for Xe III and Rn III, respectively. In Table 5, electric dipole (E1) transition parameters between some high levels for $\mathrm{Kr}$ III and only one transition for Xe III have been presented, and compared with those from computational and experimental works $[2,12,16$, 17, 24]. For transition probabilities, our results are mostly in good agreement with results presented by Djenize et al. [2] and Kernahan et al. [12]. There are a few electric dipole transition data for $\mathrm{Kr}$ III and Xe III; but there is no data for Rn III in literature. The most of results presented here for these ions, in particular Rn III, are new. 
In this work, we have only presented the electric dipole transition parameters from high levels to the levels of ground configuration in Table 2, Table 3, and Table 4. The results in detail have been given as supplementary data for each ion. Atomic data on energy spectra has great importance in astrophysics and plasma physics applications. Therefore, we hope that the electric dipole transition parameters obtained from this work for doubly ionized krypton, xenon, and radon will be useful for the experimental and theoretical studies in future.

\section{Acknowledgments}

The authors are very grateful to the anonymous reviewers for stimulating comments and valuable suggestions, which resulted in improving of the paper.

\section{References}

[1] J. Ekman, P. Jönsson, S. Gustafsson, H. Hartman, G. Gaigalas, M.R. Godefroid, and C. Froese Fischer. A\&A, 564, A24 (2014). doi: 10.1051/0004-6361/201323163

[2] S. Djeniže, V. Milosavljević, and M. S. Dimitrijević. Eur. Phys. J. D, 27, 209 (2003). doi:10.1140/epjd/e2003-00278-2.

[3] R. A. Walch, and R. D. Knight. Phys. Rev. A, 38(5), 2375 (1988). doi:10.1103/PhysRevA.38.2375.

[4] E. Träbert, P. Beiersdorfer, G. V. Brown, H. Chen, D. B. Thorn, and E. Biémont. Phys. Rev. A, 64, 042511 (2001). doi:10.1103/PhysRevA.64.042511.

[5] E. Träbert. Phy. Scr. 85, 048101 (2012).doi:10.1088/0031-8949/85/04/048101.

[6] A. G. Calamai, and C. E. Johnson. Phys. Rev. A45(11), 7792 (1992). doi:10.1103/PhysRevA.45.7792.

[7] E. Biémont, and J. E. Hansen. Phys. Scr. 34, 116 (1986). doi:10.1088/00318949/34/2/005.

[8] F. Bredice, J. R. Almandos, and M. Gallardo. J. Opt. Soc. Am. B, 5(2), 222 (1988). doi:10.1364/JOSAB.5.000222.

[9] S. Itoi, H. Kai, M. Saito, and Y. Haruyama. Phys. Scr. T156, 014023 (2013). doi:10.1088/0031-8949/2013/T156/014023. 
[10] M. Saito, N. Ojima, S. Itoi, and Y. Haruyama. Phys. Rev. A, 91, 012508 (2015). doi:10.1103/PhysRevA.91.012508.

[11] J. C. Boyce. Phys. Rev. 47, 718 (1935). doi:10.1103/PhysRev.47.718.

[12] J. A. Kernahan, E. H. Pinnington, and W. Ansbacher. J. Opt. Soc. Am B, 4(7), 1130 (1987). doi:10.1364/JOSAB.4.001130.

[13] C. J. Humphreys. Phys. Rev. 47, 712 (1935).doi:10.1103/PhysRev.47.712.

[14] D. E. Osterbrock. Astrophys. J. 114, 469 (1951).

[15] R. H. Garstang. J. Res. Natl. Bur. Stand Sec. A, 68A(1), 61 (1963). doi:10.6028/jres.068A.004.

[16] U. Fink, S. Bashkin, and W. S. Bickel. J. Quant. Spectrosc. Radiat. Transfer, 10, 1241 (1970). doi:10.1016/0022-4073(70)90008-7.

[17] M. Raineri, J. G. Reyna Almandos, F. Bredice, M. Gallardo, A. G. Trigueiros, and S-G Pettersson. J. Quant. Spectrosc. Radiat. Transfer, 60(1), 25 (1998). doi:10.1016/00224073(70)90008-7.

[18] E. B. Saloman. J. Phys. Chem. Ref. Data, 36(1), 215 (2007). doi:10.1063/1.2227036.

[19] R. J. Peláez, S. Djurović, M. Ćirišan, J. A. Aparicio, and S. Mar. A\&A, 539, A40 (2012). doi:10.1051/0004-6361/201118401.

[20] S. Schippers, S. Ricz, T. Buhr, A. Borovik Jr, J. Hellhund, K. Holste, K. Huber, H-J Schäfer, D. Schury, S. Klumpp, K. Mertens, M. Martins, R. Flesch, G. Ulrich, E. Rühl, T. Jahnke, J. Lower, D. Metz, L. P. H. Schmidt, M. Schöffler, J. B. Williams, L. Glaser, F. Scholz, J. Seltmann, J. Viefhaus, A. Dorn, A. Wolf, J. Ullrich, and A. Müller. J. Phys. B: At. Mol. Opt. Phys. 47, 115602 (2014). doi:10.1088/0953-4075/47/11/115602.

[21] T. Koizumi, Y. Awaya, A. Fujino, Y. Itoh, M. Kitajima, T. M. Kojima, M. Oura, R. Okuma, M. Sano, T. Seikioka, N. Watanabe, and F. Koike. Phys. Scr. T73, 131 (1997). doi:10.1088/0031-8949/1997/T73/042.

[22] E. Biémont, J. E. Hansen, P. Quinet, and C. J. Zeippen. Astron. Astrophys. Suppl. Ser. 111, 333 (1995).

[23] K. G. Bhushan, H. B. Pedersen, N. Altstein, O. Heber, M. L. Rappaport, and D. Zajfman. Phys. Rev. A, 62, 012504 (2000). Doi:10.1103/PhysRevA.62.012504.

[24] H. Sobral, M. Raineri, D. Schinca, M. Gallardo, and R. Duchowicz. IEEE J. Quantum Electron. 35(9), 1308 (1999). doi:10.1109/3.784590. 
[25] M. Gallardo, C. A. Massone, A. A. Tagliaferri, and M. Garavaglia. Phys. Scr. 19, 538 (1979). doi:10.1088/0031-8949/19/5-6/008.

[26] P. Bolognesi, S. J. Cavanagh, L. Avaldi, R. Camilloni, M. Zitnik, M. Stuhec, and G. C. King. J. Phys. B: At. Mol. Opt. Phys. 33, 4723 (2000). doi:10.1088/09534075/33/21/316.

[27] M. Pernpointner. J. Phys. B: At. Mol. Opt. Phys. 43, 205102 (2010). doi:10.1088/09534075/43/20/205102.

[28] P. Andersen, T. Andersen, F. Folkmann, V. K. Ivanov, H. Kjeldsen, and J. B. West. J. Phys. B: At. Mol. Opt. Phys. 34, 2009 (2001). doi:10.1088/0953-4075/34/10/314.

[29] E. B. Saloman. J. Phys. Chem. Ref. Data, 33(3), 765 (2004). doi:10.1063/1.1649348.

[30] E. Biémont, and P. Quinet. Phys. Scr. 54, 36 (1996). doi: 10.1088/0031-8949/54/1/006.

[31] M. Pernpointner, J. P. Zobel, and N. V. Kryzhevoi. Phys. Rev. A, 85, 012505 (2012). doi:10.1103/PhysRevA.85.012505.

[32] Norrington P. DARC, the Atomic R-matrix codes, GRASP0 manual (The Queen's University of Belfast, Northern Ireland, UK, 2002) homepage, http://web.am.qub.ac.uk/DARC/.

[33] K. G. Dyall, I. P. Grant, C. T. Johnson, F. A. Parpia, and E. P. Plummer, Comp. Phys. Commun. 55, 425 (1989). doi.org/10.1016/0010-4655(89)90136-7. 
Table 2. Transition probabilities, $A_{\mathrm{ij}}\left(\mathrm{s}^{-1}\right)$, the logarithm of the weighted oscillator strength, $\log (\mathrm{gf})$, and line strengths, $S_{\mathrm{ij}}$ (a.u.), for the electric dipole (E1) transitions between some low-lying levels of Kr III. The number in brackets represents the power of 10 .

\begin{tabular}{|c|c|c|c|c|c|c|c|c|}
\hline \multirow{2}{*}{\multicolumn{2}{|c|}{ Lower Level }} & & & \multirow{3}{*}{$\frac{\mathbf{A}_{\mathrm{ij}}\left(\mathbf{s}^{-1}\right)}{\text { This work }}$} & \multicolumn{2}{|c|}{$\log$ (gf) } & \multirow{3}{*}{$\begin{array}{l}\mathrm{S}_{\mathrm{ij}}(\mathrm{a} . \mathrm{u}) \\
\text { This } \\
\text { work } \\
6.105\end{array}$} & \multirow{3}{*}{$\begin{array}{c}\text { Ratio } \\
\text { This } \\
\text { work } \\
0.84\end{array}$} \\
\hline & & \multicolumn{2}{|c|}{ Upper Level } & & \multicolumn{2}{|c|}{$\begin{array}{l}\text { This } \\
\text { work }\end{array}$} & & \\
\hline $4 s^{2} 4 p^{4}$ & ${ }^{3} \mathrm{P}_{2}$ & $-4 s 4 p^{5}$ & ${ }^{3} \mathrm{P}_{2}^{\circ}$ & & $\overline{0.403}$ & -0.664 & & \\
\hline $4 s^{2} 4 p^{4}$ & ${ }^{3} \mathrm{P}_{2}$ & $-4 s^{2} 4 p^{3}\left({ }^{4} S^{\circ}\right) 5 s$ & ${ }^{5} \mathrm{~S}_{2}{ }_{2}$ & $1.949(7)$ & -2.106 & -1.897 & 0.019 & 0.79 \\
\hline $4 s^{2} 4 p^{4}$ & ${ }^{3} \mathrm{P}_{2}$ & $-4 s 4 p^{5}$ & ${ }^{3} \mathrm{P}_{1}^{\circ}$ & $4.384(9)$ & -0.219 & - & 2.367 & 0.84 \\
\hline $4 s^{2} 4 p^{4}$ & ${ }^{3} \mathrm{P}_{2}$ & $-4 s^{2} 4 p^{3}\left({ }^{4} S^{\circ}\right) 5 s$ & ${ }^{3} \mathrm{~S}_{1}^{\circ}$ & $3.639(9)$ & -0.323 & -0.115 & 1.814 & 0.72 \\
\hline $4 s^{2} 4 p^{4}$ & ${ }^{3} \mathrm{P}_{2}$ & $-4 s^{2} 4 p^{3}\left({ }^{2} D^{\circ}\right) 5 s$ & ${ }^{3} \mathrm{D}^{\circ}{ }_{1}$ & $7.981(7)$ & -2.074 & -1.703 & 0.028 & 0.82 \\
\hline $4 s^{2} 4 p^{4}$ & ${ }^{3} \mathrm{P}_{2}$ & $-4 s^{2} 4 p^{3}\left({ }^{2} D^{\circ}\right) 5 s$ & ${ }^{3} \mathrm{D}_{2}^{\circ}$ & $1.142(9)$ & -0.474 & - & 0.687 & 0.76 \\
\hline $4 s^{2} 4 p^{4}$ & ${ }^{3} \mathrm{P}_{2}$ & $-4 s^{2} 4 p^{3}\left({ }^{2} D^{\circ}\right) 5 s$ & ${ }^{3} \mathrm{D}_{3}^{\circ}$ & $2.010(9)$ & -0.092 & 0.215 & 1.650 & 0.75 \\
\hline $4 s^{2} 4 p^{4}$ & ${ }^{3} \mathrm{P}_{2}$ & $-4 s^{2} 4 p^{3}\left({ }^{2} D^{\circ}\right) 5 s$ & ${ }^{1} \mathrm{D}_{2}^{\circ}$ & $1.266(8)$ & -1.454 & -0.765 & 0.070 & 0.74 \\
\hline $4 s^{2} 4 p^{4}$ & ${ }^{3} \mathrm{P}_{1}$ & $-4 s 4 p^{5}$ & ${ }^{3} \mathrm{P}_{2}^{\circ}$ & $1.919(9)$ & -0.083 & -1.081 & 2.060 & 0.84 \\
\hline $4 s^{2} 4 p^{4}$ & ${ }^{3} \mathrm{P}_{1}$ & $-4 s^{2} 4 p^{3}\left({ }^{4} S^{\circ}\right) 5 s$ & ${ }^{5} \mathrm{~S}_{2}^{\circ}$ & $2.369(6)$ & -2.993 & -2.682 & 0.002 & 0.81 \\
\hline $4 s^{2} 4 p^{4}$ & ${ }^{3} \mathrm{P}_{1}$ & $-4 s 4 p^{5}$ & ${ }^{3} \mathrm{P}_{1}^{\circ}$ & $1.848(9)$ & -0.345 & -1.340 & 1.097 & 0.85 \\
\hline $4 s^{2} 4 p^{4}$ & ${ }^{3} \mathrm{P}_{1}$ & $-4 s 4 p^{5}$ & ${ }^{3} \mathrm{P}_{0}^{\circ}$ & $8.819(9)$ & -0.633 & -1.231 & 1.669 & 0.85 \\
\hline $4 s^{2} 4 p^{4}$ & ${ }^{3} \mathrm{P}_{1}$ & $-4 s^{2} 4 p^{3}\left({ }^{4} S^{\circ}\right) 5 s$ & ${ }^{3} \mathrm{~S}_{1}{ }_{1}$ & $2.254(9)$ & -0.282 & -0.481 & 1.232 & 0.73 \\
\hline $4 s^{2} 4 p^{4}$ & ${ }^{3} \mathrm{P}_{1}$ & $-4 s^{2} 4 p^{3}\left({ }^{2} D^{\circ}\right) 5 s$ & ${ }^{3} \mathrm{D}^{\circ}{ }_{1}$ & $1.277(9)$ & -0.624 & -0.841 & 0.503 & 0.76 \\
\hline $4 s^{2} 4 p^{4}$ & ${ }^{3} \mathrm{P}_{1}$ & $-4 s^{2} 4 p^{3}\left({ }^{2} D^{\circ}\right) 5 s$ & ${ }^{3} \mathrm{D}_{2}^{\circ}$ & $8.635(8)$ & -0.573 & -1.076 & 0.565 & 0.75 \\
\hline $4 s^{2} 4 p^{4}$ & ${ }^{3} \mathrm{P}_{1}$ & $-4 s^{2} 4 p^{3}\left({ }^{2} D^{\circ}\right) 5 s$ & ${ }^{1} \mathrm{D}_{2}^{\circ}$ & $2.511(8)$ & -1.133 & -1.105 & 0.151 & 0.78 \\
\hline $4 s^{2} 4 p^{4}$ & ${ }^{3} \mathrm{P}_{0}$ & $-4 s 4 p^{5}$ & ${ }^{3} \mathrm{P}_{1}^{\circ}$ & $2.471(9)$ & -0.214 & -1.217 & 1.492 & 0.86 \\
\hline $4 s^{2} 4 p^{4}$ & ${ }^{3} \mathrm{P}_{0}$ & $-4 s^{2} 4 p^{3}\left({ }^{4} S^{\circ}\right) 5 s$ & ${ }^{3} \mathrm{~S}_{1}^{\circ}$ & $9.342(8)$ & -0.660 & -0.886 & 0.519 & 0.74 \\
\hline $4 s^{2} 4 p^{4}$ & ${ }^{3} \mathrm{P}_{0}$ & $-4 s^{2} 4 p^{3}\left({ }^{2} D^{\circ}\right) 5 s$ & ${ }^{3} \mathrm{D}^{\circ}{ }_{1}$ & $7.016(8)$ & -0.879 & - & 0.280 & 0.75 \\
\hline $4 s^{2} 4 p^{4}$ & ${ }^{1} \mathrm{D}_{2}$ & $-4 s 4 p^{5}$ & ${ }^{3} \mathrm{P}_{2}^{\circ}$ & $9.979(7)$ & -1.276 & -1.971 & 0.146 & 0.80 \\
\hline $4 s^{2} 4 p^{4}$ & ${ }^{1} \mathrm{D}_{2}$ & $-4 s^{2} 4 p^{3}\left({ }^{4} S^{\circ}\right) 5 s$ & ${ }^{5} \mathrm{~S}_{2}^{\circ}$ & $5.971(4)$ & -2.743 & -3.345 & 0.000 & 0.64 \\
\hline $4 s^{2} 4 p^{4}$ & ${ }^{1} \mathrm{D}_{2}$ & $-4 s^{2} 4 p^{3}\left({ }^{4} S^{\circ}\right) 5 s$ & ${ }^{3} \mathrm{~S}_{1}^{\circ}$ & $9.731(6)$ & -3.260 & -2.535 & 0.007 & 0.63 \\
\hline $4 s^{2} 4 p^{4}$ & ${ }^{1} \mathrm{D}_{2}$ & $-4 s^{2} 4 p^{3}\left({ }^{2} D^{\circ}\right) 5 s$ & ${ }^{3} \mathrm{D}^{\circ}{ }_{1}$ & $1.577(8)$ & -1.678 & -1.562 & 0.081 & 0.77 \\
\hline $4 s^{2} 4 p^{4}$ & ${ }^{1} \mathrm{D}_{2}$ & $-4 s^{2} 4 p^{3}\left({ }^{2} D^{\circ}\right) 5 s$ & ${ }^{3} \mathrm{D}_{2}^{\circ}$ & $1.107(8)$ & -1.389 & -1.335 & 0.094 & 0.71 \\
\hline $4 s^{2} 4 p^{4}$ & ${ }^{1} \mathrm{D}_{2}$ & $-4 s^{2} 4 p^{3}\left({ }^{2} D^{\circ}\right) 5 s$ & ${ }^{3} \mathrm{D}_{3}^{\circ}$ & $5.115(7)$ & -1.586 & -1.731 & 0.059 & 0.79 \\
\hline $4 s^{2} 4 p^{4}$ & ${ }^{1} \mathrm{D}_{2}$ & $-4 s^{2} 4 p^{3}\left({ }^{2} D^{\circ}\right) 5 s$ & ${ }^{1} \mathrm{D}_{2}^{\circ}$ & $4.605(9)$ & 0.204 & 0.237 & 3.589 & 0.74 \\
\hline $4 s^{2} 4 p^{4}$ & ${ }^{1} \mathrm{~S}_{0}$ & $-4 s 4 p^{5}$ & ${ }^{3} \mathrm{P}^{\circ}{ }_{1}$ & $4.469(7)$ & -1.769 & - & 0.052 & 0.56 \\
\hline
\end{tabular}


Table 2. continued

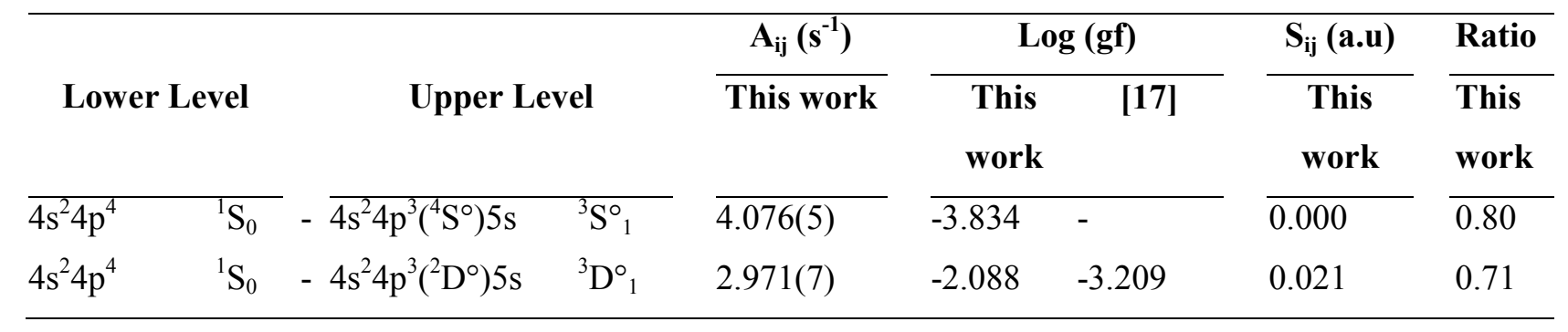


Table 3. Transition probabilities, $A_{i j}\left(s^{-1}\right)$, oscillator strengths, $\left(F_{j i}\right)$, and line strengths, $S_{i j}$ (a.u.), for the electric dipole (E1) transitions between some low-lying levels of Xe III. The number in brackets represents the power of 10 .

\begin{tabular}{|c|c|c|c|c|c|c|c|}
\hline \multicolumn{2}{|c|}{$\begin{array}{c}\text { Lower } \\
\text { Level }\end{array}$} & \multicolumn{2}{|c|}{ Upper Level } & \multirow{2}{*}{$\begin{array}{c}\mathbf{A}_{\mathrm{ij}}\left(\mathbf{s}^{-1}\right) \\
8.124(6)\end{array}$} & \multirow{2}{*}{$\begin{array}{r}\mathbf{F}_{\mathrm{ji}} \\
0.001\end{array}$} & \multirow{2}{*}{$\begin{array}{c}\begin{array}{c}S_{\mathrm{ij}} \\
\text { (a.u) }\end{array} \\
0.020\end{array}$} & \multirow{2}{*}{$\begin{array}{l}\text { Ratio } \\
0.830\end{array}$} \\
\hline $5 s^{2} 5 p^{4}$ & ${ }^{3} \mathrm{P}_{2}$ & $-5 s 5 p^{5}$ & ${ }^{3} \mathrm{P}_{2}^{\circ}$ & & & & \\
\hline $5 s^{2} 5 p^{4}$ & ${ }^{3} \mathrm{P}_{2}$ & $-\quad 5 s 5 p^{5}$ & ${ }^{3} \mathrm{P}_{1}^{\circ}$ & $3.855(6)$ & 0.000 & 0.004 & 1.400 \\
\hline $5 s^{2} 5 p^{4}$ & ${ }^{3} \mathrm{P}_{2}$ & $-\quad 5 s^{2} 5 p^{3}\left({ }^{4} S^{\circ}\right) 5 d$ & ${ }^{5} \mathrm{D}_{3}^{\circ}$ & $4.142(7)$ & 0.007 & 0.100 & 0.870 \\
\hline $5 s^{2} 5 p^{4}$ & ${ }^{3} \mathrm{P}_{2}$ & $-5 s^{2} 5 p^{3}\left({ }^{4} S^{\circ}\right) 5 d$ & ${ }^{5} \mathrm{D}_{2}^{\circ}$ & $4.082(7)$ & 0.005 & 0.069 & 0.810 \\
\hline $5 s^{2} 5 p^{4}$ & ${ }^{3} \mathrm{P}_{2}$ & $-\quad 5 s^{2} 5 p^{3}\left({ }^{4} S^{\circ}\right) 5 d$ & ${ }^{5} \mathrm{D}_{1}^{\circ}$ & $3.171(7)$ & 0.002 & 0.032 & 0.870 \\
\hline $5 s^{2} 5 p^{4}$ & ${ }^{3} \mathrm{P}_{2}$ & $-\quad 5 s^{2} 5 p^{3}\left({ }^{4} S^{\circ}\right) 5 d$ & ${ }^{3} \mathrm{D}_{2}^{\circ}$ & $9.110(7)$ & 0.009 & 0.126 & 0.880 \\
\hline $5 s^{2} 5 p^{4}$ & ${ }^{3} \mathrm{P}_{2}$ & $-\quad 5 s^{2} 5 p^{3}\left({ }^{4} S^{\circ}\right) 6 s$ & & $1.839(8)$ & 0.018 & 0.242 & 0.640 \\
\hline $5 s^{2} 5 p^{4}$ & ${ }^{3} \mathrm{P}_{0}$ & $-5 s 5 p^{5}$ & ${ }^{3} \mathrm{P}_{1}^{\circ}$ & $8.299(6)$ & 0.004 & 0.013 & 0.920 \\
\hline $5 s^{2} 5 p^{4}$ & ${ }^{3} \mathrm{P}_{0}$ & $-\quad 5 s^{2} 5 p^{3}\left({ }^{4} S^{\circ}\right) 5 d$ & ${ }^{5} \mathrm{D}_{1}^{\circ}$ & $2.212(6)$ & 0.000 & 0.002 & 0.700 \\
\hline $5 s^{2} 5 p^{4}$ & ${ }^{3} \mathrm{P}_{1}$ & $-\quad 5 s 5 p^{5}$ & ${ }^{3} \mathrm{P}_{2}^{\circ}$ & $1.040(7)$ & 0.003 & 0.034 & 0.610 \\
\hline $5 s^{2} 5 p^{4}$ & ${ }^{3} \mathrm{P}_{1}$ & $-5 s 5 p^{5}$ & ${ }^{3} \mathrm{P}_{1}^{\circ}$ & $4.380(6)$ & 0.000 & 0.007 & 0.720 \\
\hline $5 s^{2} 5 p^{4}$ & ${ }^{3} \mathrm{P}_{1}$ & $-5 s 5 p^{5}$ & ${ }^{3} \mathrm{P}_{0}^{\circ}$ & $4.928(6)$ & 0.000 & 0.002 & 1.200 \\
\hline $5 s^{2} 5 p^{4}$ & ${ }^{3} \mathrm{P}_{1}$ & $-\quad 5 s^{2} 5 p^{3}\left({ }^{4} S^{\circ}\right) 5 d$ & ${ }^{5} \mathrm{D}_{2}^{\circ}$ & $9.288(5)$ & 0.000 & 0.002 & 1.400 \\
\hline $5 s^{2} 5 p^{4}$ & ${ }^{3} \mathrm{P}_{1}$ & $-\quad 5 s^{2} 5 p^{3}\left({ }^{4} S^{\circ}\right) 5 d$ & ${ }^{5} \mathrm{D}_{1}^{\circ}$ & $1.858(7)$ & 0.002 & 0.024 & 0.820 \\
\hline $5 s^{2} 5 p^{4}$ & ${ }^{3} \mathrm{P}_{1}$ & $-\quad 5 s^{2} 5 p^{3}\left({ }^{4} S^{\circ}\right) 5 d$ & ${ }^{5} \mathrm{D}_{0}^{\circ}$ & $4.190(7)$ & 0.001 & 0.017 & 0.910 \\
\hline $5 s^{2} 5 p^{4}$ & ${ }^{3} \mathrm{P}_{1}$ & $-\quad 5 s^{2} 5 p^{3}\left({ }^{4} S^{\circ}\right) 5 d$ & ${ }^{3} \mathrm{D}_{2}^{\circ}$ & $2.465(7)$ & 0.005 & 0.043 & 0.960 \\
\hline $5 s^{2} 5 p^{4}$ & ${ }^{3} \mathrm{P}_{1}$ & $-5 s^{2} 5 p^{3}\left({ }^{4} S^{\circ}\right) 6 s$ & ${ }^{5} \mathrm{~S}_{2}^{\circ}$ & $8.460(6)$ & 0.001 & 0.014 & 0.620 \\
\hline $5 s^{2} 5 p^{4}$ & ${ }^{1} \mathrm{D}_{2}$ & $-\quad 5 s 5 p^{5}$ & ${ }^{3} \mathrm{P}_{2}^{\circ}$ & $3.118(6)$ & 0.000 & 0.014 & 0.440 \\
\hline $5 s^{2} 5 p^{4}$ & ${ }^{1} \mathrm{D}_{2}$ & $-5 s 5 p^{5}$ & ${ }^{3} \mathrm{P}^{\circ}{ }_{1}$ & $5.293(6)$ & 0.000 & 0.012 & 0.620 \\
\hline $5 s^{2} 5 p^{4}$ & ${ }^{1} \mathrm{D}_{2}$ & $-\quad 5 s^{2} 5 p^{3}\left({ }^{4} S^{\circ}\right) 5 d$ & ${ }^{5} \mathrm{D}_{3}^{\circ}$ & $1.379(5)$ & 0.000 & 0.000 & 1.300 \\
\hline $5 s^{2} 5 p^{4}$ & ${ }^{1} \mathrm{D}_{2}$ & $-\quad 5 s^{2} 5 p^{3}\left({ }^{4} S^{\circ}\right) 5 d$ & ${ }^{5} \mathrm{D}_{2}^{\circ}$ & $8.164(5)$ & 0.000 & 0.002 & 0.630 \\
\hline $5 s^{2} 5 p^{4}$ & ${ }^{1} \mathrm{D}_{2}$ & $-5 s^{2} 5 p^{3}\left({ }^{4} S^{\circ}\right) 5 d$ & ${ }^{5} \mathrm{D}^{\circ}{ }_{1}$ & $5.223(5)$ & 0.000 & 0.000 & 0.420 \\
\hline $5 s^{2} 5 p^{4}$ & ${ }^{1} \mathrm{D}_{2}$ & $-5 s^{2} 5 p^{3}\left({ }^{4} S^{\circ}\right) 5 d$ & ${ }^{3} \mathrm{D}_{2}^{\circ}$ & $1.142(5)$ & 0.000 & 0.000 & 0.001 \\
\hline $5 s^{2} 5 p^{4}$ & ${ }^{1} \mathrm{D}_{2}$ & $-\quad 5 s^{2} 5 p^{3}\left({ }^{4} S^{\circ}\right) 6 s$ & ${ }^{5} \mathrm{~S}_{2}^{\circ}$ & $7.737(3)$ & 0.000 & 0.000 & 0.460 \\
\hline $5 s^{2} 5 p^{4}$ & ${ }^{1} \mathrm{~S}_{0}$ & $-\quad 5 s 5 p^{5}$ & ${ }^{3} \mathrm{P}^{\circ}$ & $1.185(6)$ & 0.001 & 0.006 & 0.062 \\
\hline $5 s^{2} 5 p^{4}$ & ${ }^{1} \mathrm{~S}_{0}$ & $-\quad 5 s^{2} 5 p^{3}\left({ }^{4} S^{\circ}\right) 5 d$ & ${ }^{5} \mathrm{D}^{\circ}{ }_{1}$ & $2.314(5)$ & 0.000 & 0.000 & 0.120 \\
\hline
\end{tabular}


Table 4. Transition probabilities, $A_{i j}\left(s^{-1}\right)$, oscillator strengths, $\left(F_{j i}\right)$, and line strengths, $S_{i j}(a . u$.$) , for the$ electric dipole (E1) transitions between some low-lying levels of Rn III. The number in brackets represents the power of 10 .

\begin{tabular}{|c|c|c|c|c|c|c|c|}
\hline \multicolumn{2}{|c|}{ Lower Level } & \multicolumn{2}{|c|}{ Upper Level } & \multirow{2}{*}{$\begin{array}{c}\mathbf{A}_{\mathbf{i j}}\left(\mathbf{s}^{-\mathbf{1}}\right) \\
9.971(8)\end{array}$} & \multirow{2}{*}{$\begin{array}{r}\mathbf{F}_{\mathbf{j i}} \\
0.164\end{array}$} & \multirow{2}{*}{$\begin{array}{l}\mathbf{S}_{\mathbf{i j}} \text { (a.u) } \\
2.817\end{array}$} & \multirow{2}{*}{$\begin{array}{l}\text { Ratio } \\
0.88\end{array}$} \\
\hline $6 s^{2} 6 p^{4}$ & ${ }^{3} \mathrm{P}_{2}$ & $-6 s^{2} 6 p^{3}\left({ }^{4} S^{\circ}\right) 6 d$ & ${ }^{5} \mathrm{D}_{2}^{\circ}$ & & & & \\
\hline $6 s^{2} 6 p^{4}$ & ${ }^{3} \mathrm{P}_{2}$ & $-\quad 6 s^{2} 6 p^{3}\left({ }^{4} S^{\circ}\right) 6 d$ & ${ }^{5} \mathrm{D}_{2}^{\circ}$ & $3.643(7)$ & 0.006 & 0.096 & 0.41 \\
\hline $6 s^{2} 6 p^{4}$ & ${ }^{3} \mathrm{P}_{2}$ & $-\quad 6 s^{2} 6 p^{3}\left({ }^{4} S^{\circ}\right) 7 s$ & ${ }^{3} \mathrm{~S}_{1}^{\circ}$ & $1.680(9)$ & 0.158 & 2.663 & 0.88 \\
\hline $6 s^{2} 6 p^{4}$ & ${ }^{3} \mathrm{P}_{2}$ & $-6 s^{2} 6 p^{3}\left({ }^{4} S^{\circ}\right) 6 d$ & ${ }^{5} \mathrm{D}_{3}^{\circ}$ & $2.469(8)$ & 0.053 & 0.879 & 0.66 \\
\hline $6 s^{2} 6 p^{4}$ & ${ }^{3} \mathrm{P}_{2}$ & $-6 s^{2} 6 p^{3}\left({ }^{4} S^{\circ}\right) 6 d$ & ${ }^{5} \mathrm{D}^{\circ}{ }_{1}$ & $2.203(8)$ & 0.020 & 0.327 & 1.40 \\
\hline $6 s^{2} 6 p^{4}$ & ${ }^{3} \mathrm{P}_{2}$ & $-6 s^{2} 6 p^{3}\left({ }^{2} P^{\circ}\right) 6 d$ & ${ }^{3} \mathrm{P}_{2}^{\circ}$ & $5.327(8)$ & 0.078 & 1.273 & 0.63 \\
\hline $6 s^{2} 6 p^{4}$ & ${ }^{3} \mathrm{P}_{2}$ & $-6 s^{2} 6 p^{3}\left(D^{\circ}\right) 6 d$ & ${ }^{3} \mathrm{G}_{3}^{\circ}$ & $4.060(9)$ & 0.640 & 9.126 & 0.63 \\
\hline $6 s^{2} 6 p^{4}$ & ${ }^{3} \mathrm{P}_{2}$ & $-6 s^{2} 6 p^{3}\left({ }^{4} S^{\circ}\right) 6 d$ & ${ }^{3} \mathrm{D}^{\circ}{ }_{1}$ & $1.290(9)$ & 0.085 & 1.207 & 0.80 \\
\hline $6 s^{2} 6 p^{4}$ & ${ }^{3} \mathrm{P}_{0}$ & $-\quad 6 s^{2} 6 p^{3}\left({ }^{4} S^{\circ}\right) 6 d$ & ${ }^{5} \mathrm{D}_{1}^{\circ}$ & $1.176(8)$ & 0.067 & 0.248 & 0.85 \\
\hline $6 s^{2} 6 p^{4}$ & ${ }^{3} \mathrm{P}_{0}$ & $-\quad 6 s^{2} 6 p^{3}\left({ }^{4} S^{\circ}\right) 6 d$ & & $2.701(9)$ & 1.091 & 3.403 & 0.60 \\
\hline $6 s^{2} 6 p^{4}$ & ${ }^{3} \mathrm{P}_{1}$ & $-6 s^{2} 6 p^{3}\left({ }^{4} S^{\circ}\right) 6 d$ & & $1.271(6)$ & 0.000 & 0.011 & 2.40 \\
\hline $6 s^{2} 6 p^{4}$ & ${ }^{3} \mathrm{P}_{1}$ & $-6 s^{2} 6 p^{3}\left({ }^{4} S^{\circ}\right) 6 d$ & ${ }^{5} \mathrm{D}_{2}^{\circ}$ & $4.086(5)$ & 0.000 & 0.003 & 0.67 \\
\hline $6 s^{2} 6 p^{4}$ & ${ }^{3} \mathrm{P}_{1}$ & $-\quad 6 s^{2} 6 p^{3}\left({ }^{4} S^{\circ}\right) 7 s$ & ${ }^{3} \mathrm{~S}_{1}^{\circ}$ & $3.081(6)$ & 0.001 & 0.015 & 1.80 \\
\hline $6 s^{2} 6 p^{4}$ & ${ }^{3} \mathrm{P}_{1}$ & $-6 s^{2} 6 p^{3}\left({ }^{4} S^{\circ}\right) 6 d$ & ${ }^{5} \mathrm{D}^{\circ}{ }_{1}$ & $2.723(7)$ & 0.008 & 0.120 & 1.10 \\
\hline $6 s^{2} 6 p^{4}$ & ${ }^{3} \mathrm{P}_{1}$ & $-6 s^{2} 6 p^{3}\left({ }^{4} S^{\circ}\right) 6 d$ & ${ }^{5} \mathrm{D}_{0}^{\circ}$ & $1.190(7)$ & 0.001 & 0.017 & 0.51 \\
\hline $6 s^{2} 6 p^{4}$ & ${ }^{3} \mathrm{P}_{1}$ & $-6 s^{2} 6 p^{3}\left({ }^{2} P^{\circ}\right) 6 d$ & ${ }^{3} \mathrm{P}_{2}^{\circ}$ & $8.049(6)$ & 0.004 & 0.056 & 0.26 \\
\hline $6 s^{2} 6 p^{4}$ & ${ }^{1} \mathrm{D}_{2}$ & $-6 s^{2} 6 p^{3}\left({ }^{4} S^{\circ}\right) 6 d$ & ${ }^{5} \mathrm{D}_{2}^{\circ}$ & $2.092(5)$ & 0.000 & 0.003 & 0.39 \\
\hline $6 s^{2} 6 p^{4}$ & ${ }^{1} \mathrm{D}_{2}$ & $-6 s^{2} 6 p^{3}\left({ }^{4} S^{\circ}\right) 6 d$ & ${ }^{5} \mathrm{D}_{3}^{\circ}$ & $1.130(5)$ & 0.000 & 0.002 & 2.00 \\
\hline $6 s^{2} 6 p^{4}$ & ${ }^{1} \mathrm{D}_{2}$ & $-6 s^{2} 6 p^{3}\left({ }^{4} s^{\circ}\right) 6 d$ & ${ }^{5} \mathrm{D}^{\circ}{ }_{1}$ & $5.329(5)$ & 0.000 & 0.003 & 0.58 \\
\hline $6 s^{2} 6 p^{4}$ & ${ }^{1} \mathrm{D}_{2}$ & $-6 s^{2} 6 p^{3}\left({ }^{2} P^{\circ}\right) 6 d$ & ${ }^{3} \mathrm{P}_{2}^{\circ}$ & $8.851(5)$ & 0.000 & 0.009 & 0.03 \\
\hline $6 s^{2} 6 p^{4}$ & ${ }^{1} \mathrm{D}_{2}$ & $-6 s^{2} 6 p^{3}\left({ }^{2} D^{\circ}\right) 6 d$ & ${ }^{3} \mathrm{G}_{3}^{\circ}$ & $2.605(6)$ & 0.000 & 0.020 & 0.76 \\
\hline $6 s^{2} 6 p^{4}$ & ${ }^{1} \mathrm{D}_{2}$ & $-6 s^{2} 6 p^{3}\left({ }^{4} s^{\circ}\right) 6 d$ & ${ }^{3} \mathrm{D}^{\circ}{ }_{1}$ & $4.956(6)$ & 0.000 & 0.016 & 2.10 \\
\hline
\end{tabular}


Table 5. Transition probabilities, $A_{\mathrm{ij}}\left(\mathrm{s}^{-1}\right)$, logarithm of the weighted oscillator strength, Log (gf), and line strengths, $S_{\mathrm{ij}}$ (a.u.), for the electric dipole (E1) transitions for some high levels in Kr III and Xe III. The number in brackets represents the power of 10 .

\begin{tabular}{|c|c|c|c|c|c|c|c|}
\hline \multirow{2}{*}{ Lower Level } & \multirow[b]{2}{*}{ Upper Level } & \multicolumn{2}{|c|}{$\mathbf{A}_{\mathrm{ij}}\left(\mathrm{s}^{-1}\right)$} & \multicolumn{2}{|c|}{$\log ($ gf) } & \multirow{2}{*}{$\begin{array}{c}\frac{\mathrm{S}_{\mathrm{ij}}(\mathrm{a.u})}{\text { This }} \\
\text { work }\end{array}$} & \multirow{2}{*}{$\begin{array}{c}\text { Ratio } \\
\text { This } \\
\text { work }\end{array}$} \\
\hline & & $\begin{array}{c}\text { This } \\
\text { work }\end{array}$ & $\begin{array}{l}\text { Other } \\
\text { works }\end{array}$ & $\begin{array}{c}\text { This } \\
\text { work }\end{array}$ & $\begin{array}{l}\text { Other } \\
\text { work }\end{array}$ & & \\
\hline \multicolumn{8}{|l|}{ Kr III } \\
\hline \multirow[t]{4}{*}{$4 s^{2} 4 p^{3}\left(S^{4}\right) 5 s$} & $-4 s^{2} 4 p^{3}\left({ }^{4} S^{\circ}\right) 5 p \quad{ }^{3} P_{2}$ & $1.93(8)$ & $0.75(8)^{a}$ & 0.288 & $0.289^{a}$ & 23.372 & 1.0 \\
\hline & & & $1.16(8)^{b}$ & & & & \\
\hline & & & $0.85(8)^{c}$ & & & & \\
\hline & & & $1.22(8)^{d}$ & & & & \\
\hline \multirow[t]{4}{*}{$4 s^{2} 4 p^{3}\left({ }^{2} D^{\circ}\right) 5 s \quad{ }^{3} D^{\circ}{ }_{1}$} & $-4 s^{2} 4 p^{3}\left({ }^{2} D^{\circ}\right) 5 p \quad{ }^{3} F_{2}$ & $2.11(8)$ & $0.08(8)^{a}$ & 0.289 & $0.202^{a}$ & 22.504 & 0.90 \\
\hline & & & $1.10(8)^{b}$ & & & & \\
\hline & & & $0.97(8)^{c}$ & & & & \\
\hline & & & $1.59(8)^{d}$ & & & & \\
\hline \multirow[t]{4}{*}{$4 s^{2} 4 p^{3}\left({ }^{4} S^{\circ}\right) 5 s \quad{ }^{5} S^{\circ}{ }_{2}$} & $-4 s^{2} 4 p^{3}\left({ }^{4} S^{\circ}\right) 5 p \quad{ }^{5} P_{1}$ & $2.31(8)$ & $0.89(8)^{a}$ & -0.118 & $0.099^{a}$ & 14.587 & 0.89 \\
\hline & & & $0.86(8)^{b}$ & & & & \\
\hline & & & $1.11(8)^{c}$ & & & & \\
\hline & & & $0.94(8)^{d}$ & & & & \\
\hline \multirow[t]{4}{*}{$4 s^{2} 4 p^{3}\left({ }^{4} S^{\circ}\right) 5 s \quad{ }^{5} S^{\circ}{ }_{2}$} & $-4 s^{2} 4 p^{3}\left({ }^{4} S^{\circ}\right) 5 p \quad{ }^{5} P_{2}$ & $2.34(8)$ & $2.80(8)^{a}$ & 0.323 & $0.313^{a}$ & 23.959 & 0.89 \\
\hline & & & $1.59(8)^{b}$ & & & & \\
\hline & & & $3.33(8)^{c}$ & & & & \\
\hline & & & $0.98(8)^{d}$ & & & & \\
\hline \multirow[t]{4}{*}{$4 s^{2} 4 p^{3}\left({ }^{2} D^{\circ}\right) 5 s^{3} D^{\circ}{ }_{2}$} & $-4 s^{2} 4 p^{3}\left({ }^{2} D^{\circ}\right) 5 p \quad{ }^{3} D_{2}$ & $1.58(8)$ & $1.13(8)^{a}$ & 0.204 & $0.157^{a}$ & 19.355 & 0.98 \\
\hline & & & $1.34(8)^{b}$ & & & & \\
\hline & & & $0.86(8)^{c}$ & & & & \\
\hline & & & $1.68(8)^{d}$ & & & & \\
\hline \multirow[t]{4}{*}{$4 s^{2} 4 p^{3}\left({ }^{2} D^{\circ}\right) 5 s \quad{ }^{3} D^{\circ}{ }_{3}$} & $-4 s^{2} 4 p^{3}\left({ }^{2} D^{\circ}\right) 5 p \quad{ }^{3} F_{4}$ & $2.35(8)$ & $0.88(8)^{a}$ & 0.590 & $0.549^{a}$ & 44.860 & 0.92 \\
\hline & & & $0.92(8)^{b}$ & & & & \\
\hline & & & $0.92(8)^{c}$ & & & & \\
\hline & & & $1.60(8)^{d}$ & & & & \\
\hline $4 s^{2} 4 p^{3}\left({ }^{4} S^{\circ}\right) 5 s \quad{ }^{5} S^{\circ}{ }_{2}$ & $-4 s^{2} 4 p^{3}\left({ }^{4} S^{\circ}\right) 5 p{ }^{5} P_{3}$ & $2.55(8)$ & $1.00(8)^{a-d}$ & 0.487 & $0.490^{a}$ & 34.238 & 0.89 \\
\hline $4 s^{2} 4 p^{3}\left({ }^{2} D^{\circ}\right) 5 s \quad{ }^{3} D^{\circ}{ }_{3}$ & $-4 s^{2} 4 p^{3}\left({ }^{2} D^{\circ}\right) 5 p \quad{ }^{3} P_{2}$ & $2.59(8)$ & $\begin{array}{l}0.80(8)^{a} \\
1.32(8)^{c} \\
1.02(8)^{d}\end{array}$ & 0.121 & $0.186^{a}$ & 18.822 & 0.86 \\
\hline
\end{tabular}


Table 5. continued

\begin{tabular}{|c|c|c|c|c|c|c|c|}
\hline \multirow[b]{2}{*}{ Lower Level } & \multirow[b]{2}{*}{ Upper Level } & \multicolumn{2}{|c|}{$\mathbf{A}_{\mathrm{ij}}\left(\mathbf{s}^{-1}\right)$} & \multicolumn{2}{|c|}{$\log ($ gf) } & \multirow{2}{*}{$\begin{array}{c}\mathrm{S}_{\mathrm{ij}}(\mathrm{a} . \mathrm{u}) \\
\text { This } \\
\text { work }\end{array}$} & \multirow{2}{*}{$\begin{array}{c}\text { Ratio } \\
\text { This } \\
\text { work }\end{array}$} \\
\hline & & $\begin{array}{c}\text { This } \\
\text { work }\end{array}$ & $\begin{array}{l}\text { Other } \\
\text { works }\end{array}$ & $\begin{array}{c}\text { This } \\
\text { work }\end{array}$ & $\begin{array}{l}\text { Other } \\
\text { work }\end{array}$ & & \\
\hline $4 \mathrm{~s}^{2} 4 \mathrm{p}^{3}\left({ }^{2} \mathrm{D}^{\circ}\right) 5 \mathrm{~s} \quad{ }^{3} \mathrm{D}_{2}^{\circ}$ & $-\overline{4 s^{2} 4 p^{3}\left({ }^{4} S^{\circ}\right) 5 p} \quad{ }^{3} P_{1}$ & $2.57(5)$ & - & -2.216 & $-1.839^{a}$ & 0.312 & 2.1 \\
\hline $4 s^{2} 4 p^{3}\left({ }^{2} D^{\circ}\right) 5 s \quad{ }^{3} D^{\circ}{ }_{1}$ & $-4 s^{2} 4 p^{3}\left({ }^{4} S^{\circ}\right) 5 p \quad{ }^{3} P_{1}$ & $2.20(5)$ & - & -2.077 & $-1.528^{a}$ & 0.254 & 2.2 \\
\hline $4 s^{2} 4 p^{3}\left({ }^{2} D^{\circ}\right) 5 s \quad{ }^{3} D^{\circ}{ }_{2}$ & $-4 s^{2} 4 p^{3}\left({ }^{4} S^{\circ}\right) 5 p \quad{ }^{3} P_{2}$ & $2.67(5)$ & - & -1.784 & $-1.497^{a}$ & 0.490 & 2.3 \\
\hline $4 \mathrm{~s}^{2} 4 \mathrm{p}^{3}\left({ }^{2} \mathrm{P}^{\circ}\right) 5 \mathrm{~s} \quad{ }^{1} \mathrm{P}_{1}^{\circ}$ & $-4 s^{2} 4 p^{3}\left({ }^{2} D^{\circ}\right) 5 p{ }^{3} P_{0}$ & $7.22(5)$ & - & -2.966 & $-2.009^{a}$ & 0.058 & 3.1 \\
\hline $4 s^{2} 4 p^{3}\left({ }^{2} D^{\circ}\right) 5 s \quad{ }^{3} D^{\circ}{ }_{1}$ & $-4 s^{2} 4 p^{3}\left({ }^{4} S^{\circ}\right) 5 p \quad{ }^{3} P_{0}$ & $8.92(5)$ & - & -2.461 & $-1.588^{a}$ & 0.301 & 2.5 \\
\hline $4 s^{2} 4 p^{3}\left({ }^{2} D^{\circ}\right) 5 s \quad{ }^{1} D^{\circ}{ }_{2}$ & $-4 s^{2} 4 p^{3}\left({ }^{2} D^{\circ}\right) 5 p \quad{ }^{3} D_{1}$ & $2.35(7)$ & - & -0.849 & $-1.311^{a}$ & 3.665 & 1.5 \\
\hline $4 s^{2} 4 p^{3}\left({ }^{2} \mathrm{P}^{\circ}\right) 5 \mathrm{~s} \quad{ }^{3} \mathrm{P}_{1}^{\circ}$ & $-4 s^{2} 4 p^{3}\left({ }^{2} D^{\circ}\right) 5 p \quad{ }^{3} P_{2}$ & $8.95(5)$ & - & -1.491 & $-0.723^{a}$ & 0.207 & 2.6 \\
\hline $4 s^{2} 4 p^{3}\left({ }^{2} D^{\circ}\right) 5 s \quad{ }^{1} D^{\circ}{ }_{2}$ & $-4 s^{2} 4 p^{3}\left({ }^{2} D^{\circ}\right) 5 p \quad{ }^{3} D_{2}$ & $2.52(6)$ & - & -1.444 & $-2.491^{a}$ & 0.516 & 1.3 \\
\hline $4 s^{2} 4 p^{3}\left({ }^{2} D^{\circ}\right) 5 s \quad{ }^{1} D^{\circ}{ }_{2}$ & $-4 s^{2} 4 p^{3}\left({ }^{2} D^{\circ}\right) 5 p \quad{ }^{1} P_{1}$ & $9.02(7)$ & - & -0.387 & $-0.950^{a}$ & 9.243 & 1.2 \\
\hline $4 s^{2} 4 p^{3}\left({ }^{4} S^{\circ}\right) 5 s \quad{ }^{3} S^{\circ}{ }_{1}$ & $-4 s^{2} 4 p^{3}\left({ }^{4} S^{\circ}\right) 5 p \quad{ }^{5} P_{1}$ & $9.46(5)$ & - & -2.035 & $-1.698^{a}$ & 0.141 & 1.2 \\
\hline $4 s^{2} 4 p^{3}\left({ }^{2} D^{\circ}\right) 5 s \quad{ }^{1} D^{\circ}{ }_{2}$ & $-4 s^{2} 4 p^{3}\left({ }^{2} D^{\circ}\right) 5 p \quad{ }^{3} D_{3}$ & $3.21(6)$ & - & -1.269 & $-0.393^{a}$ & 0.709 & 0.88 \\
\hline $4 s^{2} 4 p^{3}\left({ }^{4} S^{\circ}\right) 5 s \quad{ }^{3} S^{\circ}{ }_{1}$ & $-4 s^{2} 4 p^{3}\left({ }^{4} S^{\circ}\right) 5 p \quad{ }^{5} \mathrm{P}_{2}$ & $2.37(6)$ & - & -1.203 & $-1.089^{a}$ & 0.569 & 1.1 \\
\hline $4 s^{2} 4 p^{3}\left({ }^{2} D^{\circ}\right) 5 s \quad{ }^{1} D^{\circ}{ }_{2}$ & $-4 s^{2} 4 p^{3}\left({ }^{2} D^{\circ}\right) 5 p \quad{ }^{1} F_{3}$ & $1.72(8)$ & - & 0.438 & $-0.095^{a}$ & 35.077 & 1.0 \\
\hline $4 s^{2} 4 p^{3}\left({ }^{2} \mathrm{P}^{\circ}\right) 5 \mathrm{p} \quad{ }^{3} \mathrm{D}_{3}$ & $-4 s^{2} 4 p^{3}\left({ }^{2} D^{\circ}\right) 6 s \quad{ }^{3} D_{3}^{\circ}$ & $5.48(5)$ & - & -1.856 & $-2.166^{a}$ & 0.226 & 2.8 \\
\hline $4 \mathrm{~s}^{2} 4 \mathrm{p}^{3}\left({ }^{2} \mathrm{P}^{\circ}\right) 5 \mathrm{~s} \quad{ }^{1} \mathrm{P}_{1}^{\circ}$ & $-4 s^{2} 4 p^{3}\left({ }^{2} \mathrm{P}^{\circ}\right) 5 \mathrm{p} \quad{ }^{3} \mathrm{D}_{2}$ & $3.08(6)$ & - & 1.575 & $-1.335^{a}$ & 0.297 & 0.84 \\
\hline $4 \mathrm{~s}^{2} 4 \mathrm{p}^{3}\left({ }^{2} \mathrm{P}^{\circ}\right) 5 \mathrm{~s} \quad{ }^{3} \mathrm{P}_{0}^{\circ}$ & $-4 s^{2} 4 p^{3}\left({ }^{2} \mathrm{P}^{\circ}\right) 5 \mathrm{p} \quad{ }^{3} \mathrm{D}_{1}$ & $1.47(6)$ & 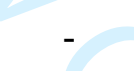 & -2.371 & $-2.472^{a}$ & 0.077 & 0.80 \\
\hline $4 s^{2} 4 p^{3}\left({ }^{2} \mathrm{P}^{\circ}\right) 5 \mathrm{~s} \quad{ }^{1} \mathrm{P}^{\circ}{ }_{1}$ & $-4 s^{2} 4 p^{3}\left({ }^{2} \mathrm{P}^{\circ}\right) 5 \mathrm{p} \quad{ }^{3} \mathrm{P}_{1}$ & $2.19(8)$ & - & -0.052 & $-0.767^{a}$ & 8.757 & 0.62 \\
\hline $4 s^{2} 4 p^{3}\left({ }^{2} D^{\circ}\right) 5 s \quad{ }^{3} D^{\circ}{ }_{2}$ & $-4 s^{2} 4 p^{3}\left({ }^{2} D^{\circ}\right) 5 p \quad{ }^{3} D_{1}$ & $5.17(7)$ & - & -0.667 & $-0.232^{a}$ & 4.634 & 0.99 \\
\hline $4 s^{2} 4 p^{3}\left({ }^{2} D^{\circ}\right) 5 s \quad{ }^{1} D^{\circ}{ }_{2}$ & $-4 s^{2} 4 p^{3}\left({ }^{2} \mathrm{P}^{\circ}\right) 5 \mathrm{p} \quad{ }^{3} \mathrm{P}_{2}$ & $1.27(7)$ & - & -1.341 & $-1.240^{a}$ & 0.328 & 0.70 \\
\hline $4 s^{2} 4 p^{3}\left({ }^{2} D^{\circ}\right) 5 s \quad{ }^{3} D^{\circ}{ }_{1}$ & $-4 s^{2} 4 p^{3}\left({ }^{2} D^{\circ}\right) 5 p \quad{ }^{3} D_{1}$ & $7.07(7)$ & - & -0.294 & $-0.261^{a}$ & 6.194 & 1.1 \\
\hline $4 \mathrm{~s}^{2} 4 \mathrm{p}^{3}\left({ }^{2} \mathrm{P}^{\circ}\right) 5 \mathrm{~s} \quad{ }^{1} \mathrm{P}_{1}^{\circ}$ & $-4 \mathrm{~s}^{2} 4 \mathrm{p}^{3}\left({ }^{2} \mathrm{P}^{\circ}\right) 5 \mathrm{p} \quad{ }^{3} \mathrm{P}_{0}$ & $6.57(6)$ & - & -2.475 & $-1.471^{a}$ & 0.106 & 1.0 \\
\hline $4 s^{2} 4 p^{3}\left({ }^{2} D^{\circ}\right) 5 s \quad{ }^{3} D^{\circ}{ }_{3}$ & $-4 s^{2} 4 p^{3}\left({ }^{2} D^{\circ}\right) 5 p \quad{ }^{3} F_{2}$ & $3.40(6)$ & - & -1.599 & $-1.338^{a}$ & 0.431 & 0.82 \\
\hline $4 s^{2} 4 p^{3}\left({ }^{2} D^{\circ}\right) 5 s \quad{ }^{1} D^{\circ}{ }_{2}$ & $-4 s^{2} 4 p^{3}\left({ }^{2} D^{\circ}\right) 5 p{ }^{3} P_{1}$ & $1.62(7)$ & - & -1.312 & $-1.728^{a}$ & 0.892 & 1.1 \\
\hline $4 s^{2} 4 p^{3}\left({ }^{2} \mathrm{P}^{\circ}\right) 5 \mathrm{p} \quad{ }^{3} \mathrm{D}_{1}$ & $-4 s^{2} 4 p^{3}\left({ }^{2} D^{\circ}\right) 6 s \quad{ }^{1} D_{2}^{\circ}$ & $8.77(5)$ & - & -1.924 & $-1.905^{a}$ & 0.167 & 2.4 \\
\hline $4 s^{2} 4 p^{3}\left({ }^{4} S^{\circ}\right) 5 s \quad{ }^{3} S^{\circ}{ }_{1}$ & $-4 s^{2} 4 p^{3}\left({ }^{4} S^{\circ}\right) 5 p \quad{ }^{3} P_{1}$ & $1.91(8)$ & - & 0.072 & $0.064^{a}$ & 14.390 & 1.1 \\
\hline $4 s^{2} 4 p^{3}\left({ }^{2} \mathrm{P}^{\circ}\right) 5 \mathrm{~s} \quad{ }^{3} \mathrm{P}_{0}^{\circ}$ & $-4 s^{2} 4 p^{3}\left({ }^{2} \mathrm{P}^{\circ}\right) 5 \mathrm{p} \quad{ }^{3} \mathrm{D}_{2}$ & $2.75(7)$ & - & -0.688 & $-1.755^{a}$ & 2.135 & 0.69 \\
\hline $4 s^{2} 4 p^{3}\left({ }^{4} S^{\circ}\right) 5 s \quad{ }^{3} S^{\circ}{ }_{1}$ & $-4 s^{2} 4 p^{3}\left({ }^{4} S^{\circ}\right) 5 p \quad{ }^{3} P_{0}$ & $2.00(8)$ & - & -0.878 & $-0.368^{a}$ & 4.758 & 1.1 \\
\hline $4 s^{2} 4 p^{3}\left({ }^{2} D^{\circ}\right) 5 s \quad{ }^{3} D^{\circ}{ }_{3}$ & $-4 s^{2} 4 p^{3}\left({ }^{2} D^{\circ}\right) 5 p \quad{ }^{3} F_{3}$ & $1.30(8)$ & - & 0.278 & $0.203^{a}$ & 23.240 & 0.99 \\
\hline $4 s^{2} 4 p^{3}\left({ }^{2} D^{\circ}\right) 5 s \quad{ }^{3} D^{\circ}{ }_{3}$ & $-4 s^{2} 4 p^{3}\left({ }^{2} D^{\circ}\right) 5 p \quad{ }^{3} D_{2}$ & $1.94(7)$ & - & -0.807 & $-0.696^{a}$ & 2.785 & 0.94 \\
\hline $4 \mathrm{~s}^{2} 4 \mathrm{p}^{3}\left({ }^{2} \mathrm{P}^{\circ}\right) 5 \mathrm{~s} \quad{ }^{3} \mathrm{P}^{\circ}{ }_{1}$ & $-4 s^{2} 4 p^{3}\left({ }^{2} \mathrm{P}^{\circ}\right) 5 \mathrm{p} \quad{ }^{3} \mathrm{D}_{1}$ & $1.62(8)$ & - & -0.164 & $-0.489^{a}$ & 6.920 & 0.64 \\
\hline
\end{tabular}


Table 5. continued

\begin{tabular}{|c|c|c|c|c|c|c|c|}
\hline \multirow[b]{2}{*}{ Lower Level } & \multirow[b]{2}{*}{ Upper Level } & \multicolumn{2}{|c|}{$\mathbf{A}_{\mathrm{ij}}\left(\mathbf{s}^{-1}\right)$} & \multicolumn{2}{|c|}{$\log ($ gf) } & \multirow{2}{*}{$\begin{array}{c}\mathrm{S}_{\mathrm{ij}}(\mathrm{a} . \mathrm{u}) \\
\text { This } \\
\text { work }\end{array}$} & \multirow{2}{*}{$\begin{array}{c}\text { Ratio } \\
\text { This } \\
\text { work }\end{array}$} \\
\hline & & $\begin{array}{c}\text { This } \\
\text { work }\end{array}$ & $\begin{array}{l}\text { Other } \\
\text { works }\end{array}$ & $\begin{array}{l}\text { This } \\
\text { work }\end{array}$ & $\begin{array}{l}\text { Other } \\
\text { work }\end{array}$ & & \\
\hline $4 \mathrm{~s}^{2} 4 \mathrm{p}^{3}\left({ }^{2} \mathrm{P}^{\circ}\right) 5 \mathrm{~s} \quad{ }^{3} \mathrm{P}_{0}^{\circ}$ & $-\overline{4 s^{2} 4 p^{3}\left({ }^{2} \mathrm{P}^{\circ}\right) 5 p} \quad{ }^{3} \mathrm{D}_{1}$ & $1.64(8)$ & - & -0.173 & $-0.369^{a}$ & 6.663 & $\overline{0.64}$ \\
\hline $4 s^{2} 4 p^{3}\left({ }^{2} \mathrm{P}^{\circ}\right) 5 \mathrm{~s} \quad{ }^{3} \mathrm{P}_{0}^{\circ}$ & $-4 \mathrm{~s}^{2} 4 \mathrm{p}^{3}\left({ }^{2} \mathrm{P}^{\circ}\right) 5 \mathrm{p} \quad{ }^{3} \mathrm{P}_{1}$ & $9.60(7)$ & - & -0.688 & $-0.830^{a}$ & 3.165 & 0.58 \\
\hline $4 s^{2} 4 p^{3}\left({ }^{2} D^{\circ}\right) 5 s \quad{ }^{3} D^{\circ}{ }_{2}$ & $-4 s^{2} 4 p^{3}\left({ }^{2} D^{\circ}\right) 5 p \quad{ }^{3} F_{2}$ & $1.20(7)$ & - & -0.952 & $-0.502^{a}$ & 1.302 & 1.1 \\
\hline $4 s^{2} 4 p^{3}\left({ }^{2} \mathrm{P}^{\circ}\right) 5 \mathrm{~s} \quad{ }^{3} \mathrm{P}_{0}^{\circ}$ & $-4 s^{2} 4 p^{3}\left({ }^{2} \mathrm{P}^{\circ}\right) 5 \mathrm{p} \quad{ }^{3} \mathrm{D}_{3}$ & $3.37(8)$ & - & 0.515 & $0.092^{a}$ & 32.800 & 0.69 \\
\hline $4 s^{2} 4 p^{3}\left({ }^{2} \mathrm{P}^{\circ}\right) 5 \mathrm{~s} \quad{ }^{1} \mathrm{P}^{\circ}{ }_{1}$ & $-4 s^{2} 4 p^{3}\left({ }^{2} \mathrm{P}^{\circ}\right) 5 p \quad{ }^{1} \mathrm{D}_{2}$ & $1.49(8)$ & - & -0.012 & $0.162^{a}$ & 9.442 & 0.75 \\
\hline $4 s^{2} 4 p^{3}\left({ }^{2} D^{\circ}\right) 5 s \quad{ }^{3} D^{\circ}{ }_{3}$ & $-4 s^{2} 4 p^{3}\left({ }^{2} D^{\circ}\right) 5 p \quad{ }^{3} D_{3}$ & $6.78(7)$ & - & -0.039 & $0.084^{a}$ & 10.779 & 0.94 \\
\hline $4 s^{2} 4 p^{3}\left({ }^{2} D^{\circ}\right) 5 s \quad{ }^{3} D^{\circ}{ }_{2}$ & $-4 s^{2} 4 p^{3}\left({ }^{2} D^{\circ}\right) 5 p \quad{ }^{3} F_{3}$ & $7.61(7)$ & - & 0.001 & $0.056^{a}$ & 11.673 & 0.91 \\
\hline $4 s^{2} 4 p^{3}\left({ }^{2} \mathrm{P}^{\circ}\right) 5 \mathrm{~s} \quad{ }^{3} \mathrm{P}_{1}^{\circ}$ & $-4 s^{2} 4 p^{3}\left({ }^{2} P^{\circ}\right) 5 p \quad{ }^{3} D_{2}$ & $3.32(8)$ & - & 0.340 & $0.078^{a}$ & 21.370 & 0.65 \\
\hline $4 s^{2} 4 p^{3}\left({ }^{2} D^{\circ}\right) 5 s \quad{ }^{3} D^{\circ}{ }_{3}$ & $-4 s^{2} 4 p^{3}\left({ }^{2} D^{\circ}\right) 5 p \quad{ }^{1} F_{3}$ & $2.47(6)$ & - & -1.499 & $-0.357^{a}$ & 0.365 & 1.2 \\
\hline $4 s^{2} 4 p^{3}\left({ }^{2} D^{\circ}\right) 5 s \quad{ }^{3} D_{2}^{\circ}$ & $-4 s^{2} 4 p^{3}\left({ }^{2} D^{\circ}\right) 5 p \quad{ }^{1} P_{1}$ & $2.26(6)$ & - & -2.129 & $-2.103^{a}$ & 0.142 & 0.97 \\
\hline $4 s^{2} 4 p^{3}\left({ }^{2} D^{\circ}\right) 5 s \quad{ }^{3} D^{\circ}{ }_{1}$ & $-4 s^{2} 4 p^{3}\left({ }^{2} D^{\circ}\right) 5 p \quad{ }^{3} D_{2}$ & $1.70(7)$ & - & -0.772 & $-0.782^{a}$ & 2.032 & 0.85 \\
\hline $4 s^{2} 4 p^{3}\left({ }^{2} \mathrm{P}^{\circ}\right) 5 \mathrm{~s} \quad{ }^{3} \mathrm{P}_{1}^{\circ}$ & $-4 s^{2} 4 p^{3}\left({ }^{2} \mathrm{P}^{\circ}\right) 5 \mathrm{p} \quad{ }^{3} \mathrm{P}_{2}$ & $4.13(6)$ & - & -1.675 & $-0.435^{a}$ & 0.182 & 0.50 \\
\hline $4 s^{2} 4 p^{3}\left({ }^{2} D^{\circ}\right) 5 s \quad{ }^{3} D^{\circ}{ }_{1}$ & $-4 s^{2} 4 p^{3}\left({ }^{2} D^{\circ}\right) 5 p \quad{ }^{1} P_{1}$ & $7.55(7)$ & - & -0.390 & $-0.258^{a}$ & 4.665 & 0.91 \\
\hline $4 s^{2} 4 p^{3}\left({ }^{2} \mathrm{P}^{\circ}\right) 5 \mathrm{~s} \quad{ }^{3} \mathrm{P}^{\circ}{ }_{1}$ & $-4 s^{2} 4 p^{3}\left({ }^{2} \mathrm{P}^{\circ}\right) 5 \mathrm{p} \quad{ }^{3} \mathrm{P}_{1}$ & $7.32(7)$ & - & -0.633 & $-0.716^{a}$ & 2.040 & 0.57 \\
\hline $4 s^{2} 4 p^{3}\left({ }^{2} \mathrm{P}^{\circ}\right) 5 \mathrm{~s} \quad{ }^{3} \mathrm{P}_{0}^{\circ}$ & $-4 s^{2} 4 p^{3}\left({ }^{2} \mathrm{P}^{\circ}\right) 5 \mathrm{p} \quad{ }^{3} \mathrm{P}_{1}$ & $1.84(7)$ & - & -1.246 & $-0.244^{a}$ & 0.490 & 0.59 \\
\hline $4 s^{2} 4 p^{3}\left({ }^{2} D^{\circ}\right) 5 s \quad{ }^{3} D^{\circ}{ }_{2}$ & $-4 s^{2} 4 p^{3}\left({ }^{2} D^{\circ}\right) 5 p \quad{ }^{3} D_{3}$ & $1.62(8)$ & & 0.296 & $-0.111^{a}$ & 22.214 & 0.87 \\
\hline $4 s^{2} 4 p^{3}\left({ }^{2} \mathrm{P}^{\circ}\right) 5 \mathrm{~s} \quad{ }^{3} \mathrm{P}^{\circ}{ }_{1}$ & $-4 s^{2} 4 p^{3}\left({ }^{2} \mathrm{P}^{\circ}\right) 5 \mathrm{p} \quad{ }^{3} \mathrm{P}_{0}$ & $4.16(8)$ & - & -0.784 & $-0.430^{a}$ & 4.567 & 0.63 \\
\hline $4 s^{2} 4 p^{3}\left({ }^{2} D^{\circ}\right) 5 s \quad{ }^{3} D^{\circ}{ }_{2}$ & $-4 s^{2} 4 p^{3}\left({ }^{2} D^{\circ}\right) 5 p \quad{ }^{1} F_{3}$ & $3.30(6)$ & - & -1.415 & $-0.810^{a}$ & 0.422 & 0.85 \\
\hline $4 s^{2} 4 p^{3}\left({ }^{2} \mathrm{P}^{\circ}\right) 5 \mathrm{~s} \quad{ }^{3} \mathrm{P}_{0}^{\circ}$ & $-4 s^{2} 4 p^{3}\left({ }^{2} \mathrm{P}^{\circ}\right) 5 \mathrm{p} \quad{ }^{1} \mathrm{D}_{2}$ & $2.31(8)$ & - & 0.123 & $-0.896^{a}$ & 12.114 & 0.62 \\
\hline $4 s^{2} 4 p^{3}\left({ }^{2} D^{\circ}\right) 5 s \quad{ }^{1} D_{2}^{\circ}$ & $-4 s^{2} 4 p^{3}\left({ }^{2} D^{\circ}\right) 5 p \quad{ }^{1} D_{2}$ & $3.94(8)$ & - & 0.385 & $0.287^{a}$ & 22.914 & 0.78 \\
\hline $4 s^{2} 4 p^{3}\left({ }^{2} \mathrm{P}^{\circ}\right) 5 \mathrm{~s} \quad{ }^{3} \mathrm{P}_{0}^{\circ}$ & $-4 s^{2} 4 p^{3}\left({ }^{2} \mathrm{P}^{\circ}\right) 5 \mathrm{p} \quad{ }^{3} \mathrm{P}_{2}$ & $1.90(8)$ & - & 0.036 & $0.216^{a}$ & 9.869 & 0.55 \\
\hline $4 s^{2} 4 p^{3}\left({ }^{2} \mathrm{P}^{\circ}\right) 5 \mathrm{~s} \quad{ }^{3} \mathrm{P}^{\circ}{ }_{1}$ & $-4 s^{2} 4 p^{3}\left({ }^{2} \mathrm{P}^{\circ}\right) 5 p \quad{ }^{1} \mathrm{D}_{2}$ & $4.69(7)$ & - & -0.617 & $-1.652^{a}$ & 2.085 & 0.63 \\
\hline $4 s^{2} 4 p^{3}\left({ }^{4} S^{\circ}\right) 5 s \quad{ }^{5} S^{\circ}{ }_{2}$ & $-4 s^{2} 4 p^{3}\left({ }^{4} S^{\circ}\right) 5 p \quad{ }^{3} P_{1}$ & $3.69(6)$ & - & -2.067 & $-1.500^{a}$ & 13.754 & 0.87 \\
\hline $4 s^{2} 4 p^{3}\left({ }^{4} S^{\circ}\right) 5 s \quad{ }^{5} S_{2}^{\circ}$ & $-4 s^{2} 4 p^{3}\left({ }^{4} S^{\circ}\right) 5 p \quad{ }^{3} P_{2}$ & $9.00(6)$ & - & -1.246 & $-0.946^{a}$ & 0.542 & 0.87 \\
\hline $4 s^{2} 4 p^{3}\left({ }^{2} D^{\circ}\right) 5 s \quad{ }^{3} D^{\circ}{ }_{2}$ & $-4 s^{2} 4 p^{3}\left({ }^{2} D^{\circ}\right) 5 p{ }^{3} P_{2}$ & $4.88(7)$ & - & -0.494 & $-0.670^{a}$ & 3.125 & 0.94 \\
\hline $4 s^{2} 4 p^{3}\left({ }^{2} D^{\circ}\right) 5 p \quad{ }^{3} P_{1}$ & $-4 s^{2} 4 p^{3}\left({ }^{2} D^{\circ}\right) 6 s \quad{ }^{3} D_{2}^{\circ}$ & $4.35(7)$ & - & -0.243 & $-0.009^{a}$ & 3.412 & 1.3 \\
\hline $4 s^{2} 4 p^{3}\left({ }^{2} \mathrm{P}^{\circ}\right) 5 \mathrm{~s} \quad{ }^{3} \mathrm{P}_{1}^{\circ}$ & $-4 s^{2} 4 p^{3}\left({ }^{2} \mathrm{P}^{\circ}\right) 5 \mathrm{p} \quad{ }^{3} \mathrm{P}_{2}$ & $4.13(6)$ & - & -1.675 & $-1.068^{a}$ & 0.182 & 0.5 \\
\hline $4 s^{2} 4 p^{3}\left({ }^{2} D^{\circ}\right) 5 s \quad{ }^{3} D^{\circ}{ }_{2}$ & $-4 s^{2} 4 p^{3}\left({ }^{2} D^{\circ}\right) 5 p{ }^{3} P_{1}$ & $2.48(8)$ & - & -0.244 & $-0.333^{a}$ & 9.129 & 0.7 \\
\hline $4 s^{2} 4 p^{3}\left({ }^{4} S^{\circ}\right) 5 p \quad{ }^{3} P_{2}$ & $-4 s^{2} 4 p^{3}\left({ }^{4} S^{\circ}\right) 6 s \quad{ }^{5} S_{2}^{\circ}$ & $4.27(6)$ & - & -1.518 & $-1.895^{a}$ & 0.308 & 0.91 \\
\hline $4 s^{2} 4 p^{3}\left({ }^{2} D^{\circ}\right) 5 s \quad{ }^{3} D^{\circ}{ }_{1}$ & $-4 s^{2} 4 p^{3}\left({ }^{2} D^{\circ}\right) 5 p{ }^{3} P_{0}$ & $3.67(8)$ & - & -0.811 & $-0.631^{a}$ & 4.427 & 0.75 \\
\hline
\end{tabular}


Table 5. continued

\begin{tabular}{|c|c|c|c|c|c|c|c|}
\hline \multirow[b]{2}{*}{ Lower Level } & \multirow[b]{2}{*}{ Upper Level } & \multicolumn{2}{|c|}{$A_{i j}\left(s^{-1}\right)$} & \multicolumn{2}{|c|}{$\log$ (gf) } & \multirow{2}{*}{$\begin{array}{c}\mathrm{S}_{\mathrm{ij}}(\mathrm{a} . \mathrm{u}) \\
\text { This } \\
\text { work }\end{array}$} & \multirow{2}{*}{$\begin{array}{c}\text { Ratio } \\
\text { This } \\
\text { work }\end{array}$} \\
\hline & & $\begin{array}{l}\text { This } \\
\text { work }\end{array}$ & $\begin{array}{l}\text { Other } \\
\text { works }\end{array}$ & $\begin{array}{l}\text { This } \\
\text { work }\end{array}$ & $\begin{array}{l}\text { Other } \\
\text { work }\end{array}$ & & \\
\hline 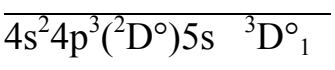 & $-\overline{4 s^{2} 4 p^{3}\left({ }^{2} D^{\circ}\right) 5 p{ }^{3} P_{1}}$ & $9.91(7)$ & - & -0.424 & $-0.697^{a}$ & 3.599 & $\overline{0.83}$ \\
\hline $4 s^{2} 4 p^{3}\left({ }^{2} \mathrm{P}^{\circ}\right) 5 p \quad{ }^{3} \mathrm{P}_{2}$ & $-4 s^{2} 4 p^{3}\left({ }^{2} \mathrm{P}^{\circ}\right) 6 \mathrm{~s} \quad{ }^{3} \mathrm{P}^{\circ}{ }_{1}$ & $1.13(5)$ & - & -3.530 & $-1.283^{a}$ & 0.005 & 5.5 \\
\hline $4 s^{2} 4 p^{3}\left({ }^{2} D^{\circ}\right) 5 s \quad{ }^{1} D^{\circ}{ }_{2}$ & $-4 s^{2} 4 p^{3}\left({ }^{2} \mathrm{P}^{\circ}\right) 5 \mathrm{p} \quad{ }^{3} \mathrm{D}_{1}$ & $1.98(7)$ & - & -1.478 & $-0.688^{a}$ & 0.456 & 0.44 \\
\hline $4 s^{2} 4 p^{3}\left({ }^{2} D^{\circ}\right) 5 p{ }^{3} P_{1}$ & $-4 s^{2} 4 p^{3}\left({ }^{2} D^{\circ}\right) 6 s \quad{ }^{1} D^{\circ}{ }_{2}$ & $1.28(7)$ & - & -1.072 & $-1.014^{a}$ & 0.831 & 1.2 \\
\hline $4 s^{2} 4 p^{3}\left({ }^{2} D^{\circ}\right) 5 p{ }^{3} P_{2}$ & $-4 s^{2} 4 p^{3}\left({ }^{2} D^{\circ}\right) 6 s \quad{ }^{3} D^{\circ}{ }_{3}$ & $8.23(7)$ & - & -0.106 & $0.006^{a}$ & 7.761 & 1.2 \\
\hline $4 s^{2} 4 p^{3}\left({ }^{2} \mathrm{P}^{\circ}\right) 5 \mathrm{p} \quad{ }^{3} \mathrm{P}_{2}$ & $-4 s^{2} 4 p^{3}\left({ }^{2} \mathrm{P}^{\circ}\right) 6 s \quad{ }^{1} \mathrm{P}_{1}^{\circ}$ & $1.54(8)$ & - & -0.396 & $-1.472^{a}$ & 5.432 & 1.1 \\
\hline $4 s^{2} 4 p^{3}\left({ }^{2} \mathrm{P}^{\circ}\right) 5 \mathrm{p} \quad{ }^{1} \mathrm{D}_{2}$ & $-4 s^{2} 4 p^{3}\left({ }^{2} P^{\circ}\right) 6 s \quad{ }^{3} P^{\circ}{ }_{1}$ & $3.49(7)$ & - & -1.042 & $-1.023^{a}$ & 1.545 & 1.3 \\
\hline $4 s^{2} 4 p^{3}\left({ }^{4} S^{\circ}\right) 5 p \quad{ }^{3} P_{2}$ & $-4 s^{2} 4 p^{3}\left({ }^{4} S^{\circ}\right) 6 s \quad{ }^{3} S^{\circ}{ }_{1}$ & $2.24(8)$ & - & -0.299 & $-0.082^{a}$ & 7.945 & 1.4 \\
\hline $4 s^{2} 4 p^{3}\left({ }^{2} D^{\circ}\right) 5 s \quad{ }^{1} D_{2}^{\circ}$ & $-4 s^{2} 4 p^{3}\left({ }^{2} P^{\circ}\right) 5 p \quad{ }^{3} D_{2}$ & $3.97(4)$ & - & -3.977 & $-1.548^{a}$ & 0.001 & 0.019 \\
\hline $4 s^{2} 4 p^{3}\left({ }^{4} S^{\circ}\right) 5 p \quad{ }^{3} P_{1}$ & $-4 s^{2} 4 p^{3}\left({ }^{4} S^{\circ}\right) 6 s \quad{ }^{3} S^{\circ}{ }_{1}$ & $1.45(8)$ & - & -0.275 & $-0.288^{a}$ & 4.988 & 1.3 \\
\hline $4 s^{2} 4 p^{3}\left({ }^{2} D^{\circ}\right) 5 s \quad{ }^{1} D^{\circ}{ }_{2}$ & $-4 s^{2} 4 p^{3}\left({ }^{2} P^{\circ}\right) 5 p \quad{ }^{3} P_{1}$ & $1.23(7)$ & - & -1.786 & $-0.844^{a}$ & 0.200 & 0.26 \\
\hline $4 s^{2} 4 p^{3}\left({ }^{2} D^{\circ}\right) 5 p{ }^{3} F_{4}$ & $-4 s^{2} 4 p^{3}\left({ }^{2} D^{\circ}\right) 6 s \quad{ }^{3} D^{\circ}{ }_{3}$ & $2.74(8)$ & - & 0.212 & $0.396^{a}$ & 18.618 & 0.84 \\
\hline $4 s^{2} 4 p^{3}\left({ }^{4} \mathrm{~S}^{\circ}\right) 5 \mathrm{p} \quad{ }^{5} \mathrm{P}_{3}$ & $-4 s^{2} 4 p^{3}\left({ }^{4} S^{\circ}\right) 6 s \quad{ }^{5} S_{2}^{\circ}$ & $3.21(8)$ & - & 0.089 & $0.212^{a}$ & 15.112 & 0.76 \\
\hline $4 s^{2} 4 p^{3}\left({ }^{2} D^{\circ}\right) 5 p \quad{ }^{1} F_{3}$ & $-4 s^{2} 4 p^{3}\left({ }^{2} D^{\circ}\right) 6 s \quad{ }^{3} D_{3}^{\circ}$ & $3.03(6)$ & - & -1.634 & $-0.762^{a}$ & 0.207 & 0.68 \\
\hline $4 s^{2} 4 p^{3}\left({ }^{2} D^{\circ}\right) 5 p \quad{ }^{1} P_{1}$ & $-4 s^{2} 4 p^{3}\left({ }^{2} D^{\circ}\right) 6 s \quad{ }^{3} D^{\circ}{ }_{2}$ & $8.93(3)$ & - & -4.314 & $-2.098^{a}$ & 0.000 & 14 \\
\hline $4 s^{2} 4 p^{3}\left({ }^{2} D^{\circ}\right) 5 s \quad{ }^{3} D^{\circ}{ }_{2}$ & $-4 s^{2} 4 p^{3}\left({ }^{2} D^{\circ}\right) 5 p \quad{ }^{1} D_{2}$ & $7.16(6)$ & - & -1.457 & $-1.527^{a}$ & 0.294 & 0.69 \\
\hline $4 s^{2} 4 p^{3}\left({ }^{2} \mathrm{P}^{\circ}\right) 5 \mathrm{p}^{3} \mathrm{D}_{3}$ & $-4 s^{2} 4 p^{3}\left({ }^{2} \mathrm{P}^{\circ}\right) 5 d^{3} P^{\circ}{ }_{2}$ & $2.99(8)$ & 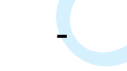 & 0.060 & $0.221^{a}$ & 14.192 & 0.83 \\
\hline $4 s^{2} 4 p^{3}\left({ }^{2} D^{\circ}\right) 5 p \quad{ }^{3} D_{2}$ & $-4 s^{2} 4 p^{3}\left({ }^{2} D^{\circ}\right) 6 s \quad{ }^{3} D_{2}^{\circ}$ & $1.66(8)$ & - & -0.078 & $-1.394^{a}$ & 7.121 & 0.82 \\
\hline $4 s^{2} 4 p^{3}\left({ }^{2} D^{\circ}\right) 5 p{ }^{3} F_{3}$ & $-4 s^{2} 4 p^{3}\left({ }^{2} D^{\circ}\right) 6 s \quad{ }^{3} D_{2}^{\circ}$ & $9.59(7)$ & - & -1.436 & $0.055^{a}$ & 4.469 & 0.84 \\
\hline $4 s^{2} 4 p^{3}\left({ }^{2} D^{\circ}\right) 5 p \quad{ }^{3} D_{3}$ & $-4 s^{2} 4 p^{3}\left({ }^{2} D^{\circ}\right) 6 s \quad{ }^{3} D_{3}^{\circ}$ & $6.78(7)$ & - & -0.300 & $-0.373^{a}$ & 4.375 & 0.82 \\
\hline $4 s^{2} 4 p^{3}\left({ }^{2} D^{\circ}\right) 5 p{ }^{1} F_{3}$ & $-4 s^{2} 4 p^{3}\left({ }^{2} D^{\circ}\right) 6 s \quad{ }^{1} D_{2}^{\circ}$ & $2.78(8)$ & - & 0.017 & $0.115^{a}$ & 12.672 & 9.6 \\
\hline $4 s^{2} 4 p^{3}\left({ }^{4} S^{\circ}\right) 5 p \quad{ }^{5} \mathrm{P}_{2}$ & $-4 s^{2} 4 p^{3}\left({ }^{4} S^{\circ}\right) 6 s \quad{ }^{5} S_{2}^{\circ}$ & $2.25(8)$ & - & 0.067 & $0.058^{a}$ & 10.085 & 0.76 \\
\hline $4 s^{2} 4 p^{3}\left({ }^{2} \mathrm{P}^{\circ}\right) 5 \mathrm{p} \quad{ }^{3} \mathrm{P}_{1}$ & $-4 s^{2} 4 p^{3}\left({ }^{2} P^{\circ}\right) 6 s \quad{ }^{3} P^{\circ}{ }_{1}$ & $9.75(6)$ & - & -0.921 & $-0.772^{a}$ & 0.409 & 0.96 \\
\hline $4 s^{2} 4 p^{3}\left({ }^{4} S^{\circ}\right) 5 p \quad{ }^{5} P_{1}$ & $-4 s^{2} 4 p^{3}\left({ }^{4} S^{\circ}\right) 6 s \quad{ }^{5} S_{2}^{\circ}$ & $1.37(8)$ & - & -0.156 & $-0.157^{a}$ & 5.996 & 0.76 \\
\hline $4 s^{2} 4 p^{3}\left({ }^{2} \mathrm{P}^{\circ}\right) 5 \mathrm{p} \quad{ }^{3} \mathrm{P}_{0}$ & $-4 s^{2} 4 p^{3}\left({ }^{2} P^{\circ}\right) 6 s \quad{ }^{1} P^{\circ}{ }_{1}$ & $7.57(6)$ & - & -1.615 & $-2.752^{a}$ & 0.213 & 0.80 \\
\hline $4 s^{2} 4 p^{3}\left({ }^{2} \mathrm{P}^{\circ}\right) 5 \mathrm{p} \quad{ }^{3} \mathrm{P}_{1}$ & $-4 s^{2} 4 p^{3}\left({ }^{2} \mathrm{P}^{\circ}\right) 5 d^{3} P^{\circ}{ }_{2}$ & $1.92(7)$ & - & -0.921 & $-0.951^{a}$ & 1.140 & 1.1 \\
\hline $4 s^{2} 4 p^{3}\left({ }^{2} \mathrm{P}^{\circ}\right) 5 \mathrm{p} \quad{ }^{3} \mathrm{P}_{1}$ & $-4 s^{2} 4 p^{3}\left({ }^{2} \mathrm{P}^{\circ}\right) 6 s \quad{ }^{1} \mathrm{P}_{1}^{\circ}$ & $1.14(8)$ & - & -0.389 & $-0.847^{a}$ & 3.792 & 1.0 \\
\hline $4 s^{2} 4 p^{3}\left({ }^{2} D^{\circ}\right) 5 p \quad{ }^{3} F_{2}$ & $-4 s^{2} 4 p^{3}\left({ }^{2} D^{\circ}\right) 6 s \quad{ }^{3} D_{2}^{\circ}$ & $1.15(7)$ & - & -1.213 & $-2.105^{a}$ & 0.537 & 0.77 \\
\hline $4 s^{2} 4 p^{3}\left({ }^{2} D^{\circ}\right) 5 p{ }^{3} D_{2}$ & $-4 s^{2} 4 p^{3}\left({ }^{2} D^{\circ}\right) 6 s \quad{ }^{3} D_{3}^{\circ}$ & $1.84(7)$ & - & -0.913 & $-0.652^{a}$ & 1.012 & 0.76 \\
\hline $4 s^{2} 4 p^{3}\left({ }^{2} D^{\circ}\right) 5 p{ }^{3} F_{3}$ & $-4 s^{2} 4 p^{3}\left({ }^{2} D^{\circ}\right) 6 s \quad{ }^{3} D^{\circ}{ }_{3}$ & $1.55(8)$ & - & 0.034 & $-0.340^{a}$ & 9.180 & 0.79 \\
\hline
\end{tabular}


Table 5. continued

\begin{tabular}{|c|c|c|c|c|c|c|c|}
\hline \multirow[b]{2}{*}{ Lower Level } & \multirow[b]{2}{*}{ Upper Level } & \multicolumn{2}{|c|}{$A_{i j}\left(s^{-1}\right)$} & \multicolumn{2}{|c|}{$\log ($ gf) } & \multirow{2}{*}{$\begin{array}{c}\mathrm{S}_{\mathrm{ij}}(\mathrm{a} . \mathrm{u}) \\
\text { This } \\
\text { work }\end{array}$} & \multirow{2}{*}{$\begin{array}{c}\text { Ratio } \\
\text { This } \\
\text { work }\end{array}$} \\
\hline & & $\begin{array}{c}\text { This } \\
\text { work }\end{array}$ & $\begin{array}{l}\text { Other } \\
\text { works }\end{array}$ & $\begin{array}{c}\text { This } \\
\text { work }\end{array}$ & $\begin{array}{l}\text { Other } \\
\text { work }\end{array}$ & & \\
\hline$\overline{4 s^{2} 4 p^{3}\left({ }^{2} \mathrm{P}^{\circ}\right) 5 p}{ }^{3} \mathrm{D}_{2}$ & $-\overline{4 s^{2} 4 p^{3}\left({ }^{2} \mathrm{P}^{\circ}\right) 5 d^{3} \mathrm{P}_{2}^{\circ}}$ & $1.77(7)$ & - & -0.035 & $-1.059^{a}$ & $\overline{0.763}$ & $\overline{0.84}$ \\
\hline $4 s^{2} 4 p^{3}\left({ }^{2} D^{\circ}\right) 5 p \quad{ }^{1} P_{1}$ & $-4 s^{2} 4 p^{3}\left({ }^{2} D^{\circ}\right) 6 s \quad{ }^{1} D_{2}^{\circ}$ & $8.56(7)$ & - & -0.379 & $-0.207^{a}$ & 3.513 & 0.87 \\
\hline $4 s^{2} 4 p^{3}\left({ }^{4} S^{\circ}\right) 5 s \quad{ }^{3} S^{\circ}{ }_{1}$ & $-4 s^{2} 4 p^{3}\left({ }^{2} D^{\circ}\right) 5 p \quad{ }^{3} F_{2}$ & $2.14(6)$ & - & -2.093 & $-2.138^{a}$ & 0.060 & 0.49 \\
\hline $4 s^{2} 4 p^{3}\left({ }^{2} \mathrm{P}^{\circ}\right) 5 \mathrm{p} \quad{ }^{3} \mathrm{D}_{3}$ & $-4 \mathrm{~s}^{2} 4 \mathrm{p}^{3}\left({ }^{2} \mathrm{P}^{\circ}\right) 6 \mathrm{~s} \quad{ }^{1} \mathrm{P}^{\circ}{ }_{1}$ & $2.82(7)$ & - & -1.307 & $-1.017^{a}$ & 0.688 & 0.76 \\
\hline $4 s^{2} 4 p^{3}\left({ }^{2} D^{\circ}\right) 5 p \quad{ }^{3} D_{2}$ & $-4 s^{2} 4 p^{3}\left({ }^{2} D^{\circ}\right) 6 s \quad{ }^{1} D_{2}^{\circ}$ & $2.02(7)$ & - & -1.037 & $-1.627^{a}$ & 0.745 & 0.67 \\
\hline $4 s^{2} 4 p^{3}\left({ }^{2} D^{\circ}\right) 5 p \quad{ }^{3} F_{3}$ & $-4 s^{2} 4 p^{3}\left({ }^{2} D^{\circ}\right) 6 s \quad{ }^{1} D_{2}^{\circ}$ & $2.97(6)$ & - & -1.993 & $-2.039^{a}$ & 0.118 & 0.86 \\
\hline $4 s^{2} 4 p^{3}\left({ }^{4} S^{\circ}\right) 5 p \quad{ }^{5} P_{2}$ & $-4 s^{2} 4 p^{3}\left({ }^{4} S^{\circ}\right) 6 s \quad{ }^{3} S^{\circ}{ }_{1}$ & $6.07(6)$ & - & -1.996 & $-1.387^{a}$ & 0.137 & 1.3 \\
\hline $4 s^{2} 4 p^{3}\left({ }^{4} S^{\circ}\right) 5 p \quad{ }^{5} P_{1}$ & $-4 s^{2} 4 p^{3}\left({ }^{4} S^{\circ}\right) 6 s \quad{ }^{3} S^{\circ}{ }_{1}$ & $1.50(6)$ & - & -2.388 & $-1.788^{a}$ & 0.033 & 1.3 \\
\hline $4 s^{2} 4 p^{3}\left({ }^{2} D^{\circ}\right) 5 p{ }^{3} F_{2}$ & $-4 s^{2} 4 p^{3}\left({ }^{2} D^{\circ}\right) 6 s \quad{ }^{3} D_{3}^{\circ}$ & $2.61(6)$ & - & -1.737 & $-0.340^{a}$ & 0.156 & 0.78 \\
\hline $4 s^{2} 4 p^{3}\left({ }^{2} \mathrm{P}^{\circ}\right) 5 \mathrm{p} \quad{ }^{3} \mathrm{D}_{1}$ & $-4 s^{2} 4 p^{3}\left({ }^{2} \mathrm{P}^{\circ}\right) 6 s \quad{ }^{3} \mathrm{P}_{1}^{\circ}$ & $9.97(7)$ & - & -0.503 & $-0.579^{a}$ & 2.735 & 0.89 \\
\hline $4 s^{2} 4 p^{3}\left({ }^{4} S^{\circ}\right) 5 s \quad{ }^{3} S^{\circ}{ }_{1}$ & $-4 s^{2} 4 p^{3}\left({ }^{2} D^{\circ}\right) 5 p \quad{ }^{3} D_{2}$ & $7.77(5)$ & - & -2.512 & $-2.073^{a}$ & 0.023 & 0.66 \\
\hline $4 s^{2} 4 p^{3}\left({ }^{2} D^{\circ}\right) 5 p{ }^{3} F_{2}$ & $-4 s^{2} 4 p^{3}\left({ }^{2} D^{\circ}\right) 6 s \quad{ }^{1} D^{\circ}{ }_{2}$ & $2.96(6)$ & - & -1.848 & $-1.585^{a}$ & 0.118 & 0.77 \\
\hline $4 s^{2} 4 p^{3}\left({ }^{4} S^{\circ}\right) 5 s \quad{ }^{3} S^{\circ}{ }_{1}$ & $-4 s^{2} 4 p^{3}\left({ }^{2} D^{\circ}\right) 5 p \quad{ }^{1} P_{1}$ & $1.42(5)$ & - & -3.500 & $-3.012^{a}$ & 0.002 & 0.20 \\
\hline $4 s^{2} 4 p^{3}\left({ }^{2} \mathrm{P}^{\circ}\right) 5 \mathrm{p} \quad{ }^{3} \mathrm{D}_{1}$ & $-4 s^{2} 4 p^{3}\left({ }^{2} \mathrm{P}^{\circ}\right) 6 \mathrm{~s} \quad{ }^{1} \mathrm{P}^{\circ}{ }_{1}$ & $9.58(5)$ & - & -2.5790 & $-2.576^{a}$ & 0.021 & 1.5 \\
\hline $4 s^{2} 4 p^{3}\left({ }^{2} D^{\circ}\right) 5 p{ }^{3} D_{1}$ & $-4 s^{2} 4 p^{3}\left({ }^{2} D^{\circ}\right) 6 s \quad{ }^{3} D_{2}^{\circ}$ & $5.60(7)$ & - & -0.5881 & $-0.584^{a}$ & 2.107 & 0.81 \\
\hline $4 s^{2} 4 p^{3}\left({ }^{2} D^{\circ}\right) 5 s \quad{ }^{3} D^{\circ}{ }_{2}$ & $-4 s^{2} 4 p^{3}\left({ }^{2} \mathrm{P}^{\circ}\right) 5 \mathrm{p} \quad{ }^{3} \mathrm{D}_{1}$ & $1.55(6)$ & 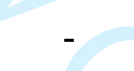 & -2.6723 & $-2.118^{a}$ & 0.026 & 0.42 \\
\hline $4 s^{2} 4 p^{3}\left({ }^{2} D^{\circ}\right) 5 p \quad{ }^{3} D_{1}$ & $-4 s^{2} 4 p^{3}\left({ }^{2} D^{\circ}\right) 6 s \quad{ }^{1} D^{\circ}{ }_{2}$ & $2.63(7)$ & - & -0.959 & $-0.992^{a}$ & 0.855 & 0.85 \\
\hline $4 s^{2} 4 p^{3}\left({ }^{2} D^{\circ}\right) 5 s \quad{ }^{3} D^{\circ}{ }_{3}$ & $-4 s^{2} 4 p^{3}\left({ }^{2} \mathrm{P}^{\circ}\right) 5 \mathrm{p} \quad{ }^{3} \mathrm{D}_{3}$ & $1.50(7)$ & - & -1.116 & $-1.372^{a}$ & 0.011 & 0.55 \\
\hline $4 s^{2} 4 p^{3}\left({ }^{2} D^{\circ}\right) 5 s \quad{ }^{3} D^{\circ}{ }_{2}$ & $-4 s^{2} 4 p^{3}\left({ }^{2} \mathrm{P}^{\circ}\right) 5 \mathrm{p} \quad{ }^{3} \mathrm{D}_{2}$ & $4.06(6)$ & - & -1.832 & $-2.122^{a}$ & 0.107 & 0.38 \\
\hline $4 s^{2} 4 p^{3}\left({ }^{2} D^{\circ}\right) 5 s \quad{ }^{3} D^{\circ}{ }_{1}$ & $-4 s^{2} 4 p^{3}\left({ }^{2} \mathrm{P}^{\circ}\right) 5 \mathrm{p} \quad{ }^{3} \mathrm{D}_{2}$ & $8.24(6)$ & - & -1.528 & $-2.584^{a}$ & 0.214 & 0.37 \\
\hline $4 s^{2} 4 p^{3}\left({ }^{2} D^{\circ}\right) 5 s \quad{ }^{3} D^{\circ}{ }_{2}$ & $-4 s^{2} 4 p^{3}\left({ }^{2} \mathrm{P}^{\circ}\right) 5 \mathrm{p} \quad{ }^{3} \mathrm{P}_{1}$ & $7.78(6)$ & - & -2.064 & $-4.560^{a}$ & 0.096 & 0.16 \\
\hline $4 s^{2} 4 p^{3}\left({ }^{2} D^{\circ}\right) 5 s \quad{ }^{3} D^{\circ}{ }_{1}$ & $-4 s^{2} 4 p^{3}\left({ }^{2} \mathrm{P}^{\circ}\right) 5 \mathrm{p} \quad{ }^{3} \mathrm{P}_{1}$ & $2.83(5)$ & - & -3.761 & $-2.157^{a}$ & 0.003 & 1.3 \\
\hline $4 s^{2} 4 p^{3}\left({ }^{2} D^{\circ}\right) 5 s \quad{ }^{3} D^{\circ}{ }_{2}$ & $-4 s^{2} 4 p^{3}\left({ }^{2} \mathrm{P}^{\circ}\right) 5 \mathrm{p} \quad{ }^{3} \mathrm{D}_{3}$ & $1.74(7)$ & - & -1.076 & $-4.372^{a}$ & 0.592 & 0.35 \\
\hline $4 s^{2} 4 p^{3}\left({ }^{2} D^{\circ}\right) 5 p \quad{ }^{1} D_{2}$ & $-4 s^{2} 4 p^{3}\left({ }^{2} \mathrm{P}^{\circ}\right) 5 d^{3} \mathrm{P}_{2}^{\circ}$ & $3.10(6)$ & - & -1.937 & $-1.593^{a}$ & 0.085 & 0.29 \\
\hline $4 s^{2} 4 p^{3}\left({ }^{4} S^{\circ}\right) 5 s \quad{ }^{3} S^{\circ}{ }_{1}$ & $-4 s^{2} 4 p^{3}\left({ }^{2} D^{\circ}\right) 5 p \quad{ }^{3} P_{2}$ & $6.93(7)$ & - & -0.683 & $-0.872^{a}$ & 1.363 & 0.55 \\
\hline $4 s^{2} 4 p^{3}\left({ }^{4} S^{\circ}\right) 5 s \quad{ }^{3} S^{\circ}{ }_{1}$ & $-4 s^{2} 4 p^{3}\left({ }^{2} D^{\circ}\right) 5 p{ }^{3} P_{0}$ & $6.93(7)$ & - & -1.868 & $-1.686^{a}$ & 0.265 & 0.57 \\
\hline $4 s^{2} 4 p^{3}\left({ }^{4} S^{\circ}\right) 5 s \quad{ }^{3} S^{\circ}{ }_{1}$ & $-4 s^{2} 4 p^{3}\left({ }^{2} D^{\circ}\right) 5 p{ }^{3} P_{1}$ & $5.19(7)$ & - & -1.039 & $-1.190^{a}$ & 0.596 & 0.58 \\
\hline $4 s^{2} 4 p^{3}\left({ }^{2} D^{\circ}\right) 5 s \quad{ }^{3} D^{\circ}{ }_{3}$ & $-4 \mathrm{~s}^{2} 4 \mathrm{p}^{3}\left({ }^{2} \mathrm{P}^{\circ}\right) 5 \mathrm{p} \quad{ }^{3} \mathrm{P}_{2}$ & $1.871(7)$ & - & -1.373 & $-2.307^{a}$ & 0.401 & 0.28 \\
\hline $4 s^{2} 4 p^{3}\left({ }^{4} S^{\circ}\right) 5 s \quad{ }^{5} S_{2}^{\circ}$ & $-4 s^{2} 4 p^{3}\left({ }^{2} D^{\circ}\right) 5 p \quad{ }^{3} F_{3}$ & $3.20(4)$ & - & -3.900 & $-2.812^{a}$ & 0.000 & 0.42 \\
\hline $4 s^{2} 4 p^{3}\left({ }^{2} D^{\circ}\right) 5 p \quad{ }^{3} P_{0}$ & $-4 s^{2} 4 p^{3}\left({ }^{2} \mathrm{P}^{\circ}\right) 6 \mathrm{~s} \quad{ }^{1} \mathrm{P}_{1}^{\circ}$ & $1.30(6)$ & - & -2.639 & $-4.254^{a}$ & 0.015 & 0.40 \\
\hline
\end{tabular}


Table 5. continued

\begin{tabular}{|c|c|c|c|c|c|c|c|c|}
\hline \multirow{2}{*}{\multicolumn{2}{|c|}{ Lower Level }} & \multirow{2}{*}{ Upper Level } & \multicolumn{2}{|c|}{$A_{i j}\left(s^{-1}\right)$} & \multicolumn{2}{|c|}{$\log ($ gf) } & \multirow{2}{*}{$\begin{array}{c}\mathrm{S}_{\mathrm{ij}}(\mathrm{a} . \mathrm{u}) \\
\text { This } \\
\text { work }\end{array}$} & \multirow{2}{*}{$\begin{array}{r}\text { Ratio } \\
\text { This } \\
\text { work }\end{array}$} \\
\hline & & & This & Other & This & Other & & \\
\hline $4 s^{2} 4 p^{3}\left({ }^{2} D^{\circ}\right) 5 p$ & ${ }^{3} \mathrm{P}_{2}$ & $-\overline{4 s^{2} 4 p^{3}\left(^{2} P^{\circ}\right) 5 d}$ & $7.54(6)$ & - & -1.649 & $-2.228^{a}$ & 0.147 & 0.68 \\
\hline $4 s^{2} 4 p^{3}\left({ }^{4} S^{\circ}\right) 5 p$ & ${ }^{3} \mathrm{P}_{2}$ & $-4 s^{2} 4 p^{3}\left({ }^{2} D^{\circ}\right) 6 s \quad{ }^{1} D^{\circ}{ }_{2}$ & $3.52(5)$ & - & -3.087 & $-3.376^{a}$ & 0.005 & 0.2 \\
\hline $4 s^{2} 4 p^{3}\left({ }^{4} S^{\circ}\right) 5 s$ & ${ }^{3} \mathrm{~S}_{1}^{\circ}$ & $-4 s^{2} 4 p^{3}\left({ }^{2} P^{\circ}\right) 5 p \quad{ }^{3} D_{2}$ & $6.90(5)$ & - & -2.867 & $-3.162^{a}$ & 0.007 & 0.27 \\
\hline $4 s^{2} 4 p^{3}\left({ }^{4} S^{\circ}\right) 5 s$ & ${ }^{3} \mathrm{~S}_{1}^{\circ}$ & $-4 s^{2} 4 p^{3}\left({ }^{2} \mathrm{P}^{\circ}\right) 5 \mathrm{p} \quad{ }^{3} \mathrm{P}_{1}$ & $8.90(5)$ & - & -3.032 & $-2.151^{a}$ & 0.005 & 1.1 \\
\hline $4 s^{2} 4 p^{3}\left({ }^{4} S^{\circ}\right) 5 s$ & ${ }^{3} \mathrm{~S}_{1}^{\circ}$ & $-4 s^{2} 4 p^{3}\left({ }^{2} \mathrm{P}^{\circ}\right) 5 \mathrm{p} \quad{ }^{3} \mathrm{P}_{0}$ & $1.07(7)$ & - & -2.402 & $-2.183^{a}$ & 0.021 & 0.68 \\
\hline $4 s^{2} 4 p^{3}\left({ }^{2} D^{\circ}\right) 5 p$ & ${ }^{3} \mathrm{D}_{1}$ & $-4 s^{2} 4 p^{3}\left({ }^{2} \mathrm{P}^{\circ}\right) 6 s \quad{ }^{3} \mathrm{P}_{1}^{\circ}$ & $1.29(6)$ & - & -2.743 & $-2.690^{a}$ & 0.010 & 0.47 \\
\hline $4 s^{2} 4 p^{3}\left({ }^{4} S^{\circ}\right) 5 p$ & ${ }^{5} \mathrm{P}_{2}$ & $-4 s^{2} 4 p^{3}\left({ }^{2} D^{\circ}\right) 6 s \quad{ }^{3} D_{3}^{\circ}$ & $5.31(5)$ & - & -2.832 & $-2.670^{a}$ & 0.007 & 0.25 \\
\hline $4 s^{2} 4 p^{3}\left({ }^{4} S^{\circ}\right) 5 s$ & ${ }^{3} \mathrm{~S}_{1}^{\circ}$ & $-4 s^{2} 4 p^{3}\left({ }^{2} \mathrm{P}^{\circ}\right) 5 \mathrm{p} \quad{ }^{1} \mathrm{D}_{2}$ & $9.41(5)$ & - & -2.793 & $-2.436^{a}$ & 0.008 & 5.1 \\
\hline $4 s^{2} 4 p^{3}\left({ }^{4} S^{\circ}\right) 5 s$ & ${ }^{3} \mathrm{~S}_{1}^{\circ}$ & $-4 \mathrm{~s}^{2} 4 \mathrm{p}^{3}\left({ }^{2} \mathrm{P}^{\circ}\right) 5 \mathrm{p} \quad{ }^{3} \mathrm{P}_{2}$ & $2.80(6)$ & - & -2.321 & $-2.353^{a}$ & 0.024 & 0.26 \\
\hline $4 s^{2} 4 p^{3}\left({ }^{4} S^{\circ}\right) 5 s$ & ${ }^{5} \mathrm{~S}_{2}$ & $-4 s^{2} 4 p^{3}\left({ }^{2} \mathrm{P}^{\circ}\right) 5 \mathrm{p} \quad{ }^{3} \mathrm{P}_{1}$ & $2.61(5)$ & - & -3.875 & $-4.776^{a}$ & 0.001 & 0.17 \\
\hline $4 s^{2} 4 p^{3}\left({ }^{4} S^{\circ}\right) 5 s$ & ${ }^{5} \mathrm{~S}_{2}^{\circ}$ & $-4 s^{2} 4 p^{3}\left({ }^{2} \mathrm{P}^{\circ}\right) 5 p \quad{ }^{1} \mathrm{D}_{2}$ & $3.81(5)$ & - & -3.275 & $-4.442^{a}$ & 0.002 & 0.13 \\
\hline $4 s^{2} 4 p^{3}\left({ }^{4} S^{\circ}\right) 5 p$ & ${ }^{3} \mathrm{P}_{2}$ & $-4 s^{2} 4 p^{3}\left({ }^{2} \mathrm{P}^{\circ}\right) 6 s \quad{ }^{3} \mathrm{P}_{1}^{\circ}$ & $2.29(6)$ & - & -2.912 & $-2.967^{a}$ & 0.009 & 0.098 \\
\hline $4 s 4 p^{5}$ & ${ }^{1} \mathrm{P}_{1}^{\circ}$ & $-4 s^{2} 4 p^{3}\left({ }^{2} \mathrm{P}^{\circ}\right) 5 \mathrm{p} \quad{ }^{1} \mathrm{D}_{2}$ & $5.21(5)$ & - & -1.773 & $-1.665^{a}$ & 0.365 & 0.60 \\
\hline $4 s 4 p^{5}$ & ${ }^{3} \mathrm{P}_{1}^{\circ}$ & $-4 s^{2} 4 p^{3}\left({ }^{2} D^{\circ}\right) 5 p \quad{ }^{3} F_{2}$ & $1.01(5)$ & - & -3.492 & $-2.473^{a}$ & 0.002 & 1.3 \\
\hline $4 s 4 p^{5}$ & ${ }^{3} \mathrm{P}_{1}^{\circ}$ & $-4 s^{2} 4 p^{3}\left({ }^{2} D^{\circ}\right) 5 p \quad{ }^{3} D_{2}$ & $1.06(5)$ & - & -3.449 & $-2.234^{a}$ & 0.002 & 0.058 \\
\hline $4 s 4 p^{5}$ & ${ }^{3} \mathrm{P}_{0}^{\circ}$ & $-4 s^{2} 4 p^{3}\left({ }^{2} D^{\circ}\right) 5 p \quad{ }^{3} P_{1}$ & $3.21(5)$ & - & -3.277 & $-1.639^{a}$ & 0.003 & 14 \\
\hline $4 s 4 p^{5}$ & ${ }^{3} \mathrm{P}_{2}^{\circ}$ & $-4 s^{2} 4 p^{3}\left({ }^{2} D^{\circ}\right) 5 p \quad{ }^{3} F_{3}$ & $3.48(5)$ & - & -2.868 & $-2.100^{a}$ & 0.008 & 0.32 \\
\hline $4 s 4 p^{5}$ & ${ }^{3} \mathrm{P}_{2}^{\circ}$ & $-4 s^{2} 4 p^{3}\left({ }^{2} D^{\circ}\right) 5 p \quad{ }^{3} D_{2}$ & $2.45(5)$ & - & -3.151 & $-2.071^{a}$ & 0.004 & 2.1 \\
\hline $4 s 4 p^{5}$ & ${ }^{3} \mathrm{P}_{2}^{\circ}$ & $-4 s^{2} 4 p^{3}\left({ }^{2} D^{\circ}\right) 5 p \quad{ }^{1} P_{1}$ & $1.04(5)$ & - & -3.992 & $-2.533^{a}$ & 0.001 & 0.047 \\
\hline $4 s 4 p^{5}$ & ${ }^{3} \mathrm{P}_{2}^{\circ}$ & $-4 s^{2} 4 p^{3}\left({ }^{2} D^{\circ}\right) 5 p \quad{ }^{3} D_{3}$ & $2.61(5)$ & - & -3.011 & $-1.701^{a}$ & 0.006 & 0.27 \\
\hline $4 s 4 p^{5}$ & ${ }^{3} \mathrm{P}_{1}^{\circ}$ & $-4 s^{2} 4 p^{3}\left({ }^{2} D^{\circ}\right) 5 p \quad{ }^{3} P_{1}$ & $7.08(5)$ & - & -2.967 & $-1.978^{a}$ & 0.006 & 3.4 \\
\hline $4 s 4 p^{5}$ & ${ }^{3} \mathrm{P}_{2}^{\circ}$ & $-4 s^{2} 4 p^{3}\left({ }^{2} D^{\circ}\right) 5 p \quad{ }^{3} P_{1}$ & $2.76(6)$ & - & -2.655 & $-1.238^{a}$ & 0.021 & 1.3 \\
\hline $4 s^{2} 4 p^{4}$ & ${ }^{1} \mathrm{~S}_{0}$ & $-4 s 4 p^{5}$ & $4.47(7)$ & - & -1.764 & $-2.738^{a}$ & 0.052 & 0.56 \\
\hline $4 s 4 p^{5}$ & ${ }^{3} \mathrm{P}_{1}^{\circ}$ & $-4 s^{2} 4 p^{3}\left({ }^{2} \mathrm{P}^{\circ}\right) 5 \mathrm{p} \quad{ }^{3} \mathrm{P}_{0}$ & $3.36(6)$ & - & -3.432 & $-2.081^{a}$ & 0.005 & 3.6 \\
\hline $4 s 4 p^{5}$ & ${ }^{3} \mathrm{P}_{2}^{\circ}$ & $-4 s^{2} 4 p^{3}\left({ }^{2} \mathrm{P}^{\circ}\right) 5 \mathrm{p} \quad{ }^{3} \mathrm{P}_{1}$ & $1.05(6)$ & - & -3.276 & $-2.036^{a}$ & 0.004 & 1.7 \\
\hline $4 s 4 p^{5}$ & ${ }^{3} \mathrm{P}_{1}^{\circ}$ & $-4 s^{2} 4 p^{3}\left({ }^{2} \mathrm{P}^{\circ}\right) 5 \mathrm{p} \quad{ }^{3} \mathrm{P}_{2}$ & $1.04(6)$ & - & -2.801 & $-2.494^{a}$ & 0.007 & 0.40 \\
\hline $4 s 4 p^{5}$ & ${ }^{3} \mathrm{P}_{2}^{\circ}$ & $-4 s^{2} 4 p^{3}\left({ }^{2} \mathrm{P}^{\circ}\right) 5 \mathrm{p} \quad{ }^{3} \mathrm{P}_{2}$ & $4.53(6)$ & - & -2.204 & $-2.840^{a}$ & 0.028 & 0.30 \\
\hline $4 s^{2} 4 p^{4}$ & ${ }^{1} \mathrm{~S}_{0}$ & $-4 s 4 p^{5}$ & $2.89(9)$ & - & -0.309 & $-2.385^{a}$ & 0.992 & 1.7 \\
\hline $4 s^{2} 4 p^{4}$ & ${ }^{1} \mathrm{D}_{2}$ & $-4 s 4 p^{5}$ & $2.60(10)$ & - & 0.350 & $-0.882^{a}$ & 6.924 & 0.49 \\
\hline $4 s^{2} 4 p^{4}$ & ${ }^{3} \mathrm{P}_{0}$ & $-4 s 4 p^{5}$ & $7.82(7)$ & - & -2.010 & $-2.644^{a}$ & 0.017 & 2.2 \\
\hline
\end{tabular}


Table 5. continued

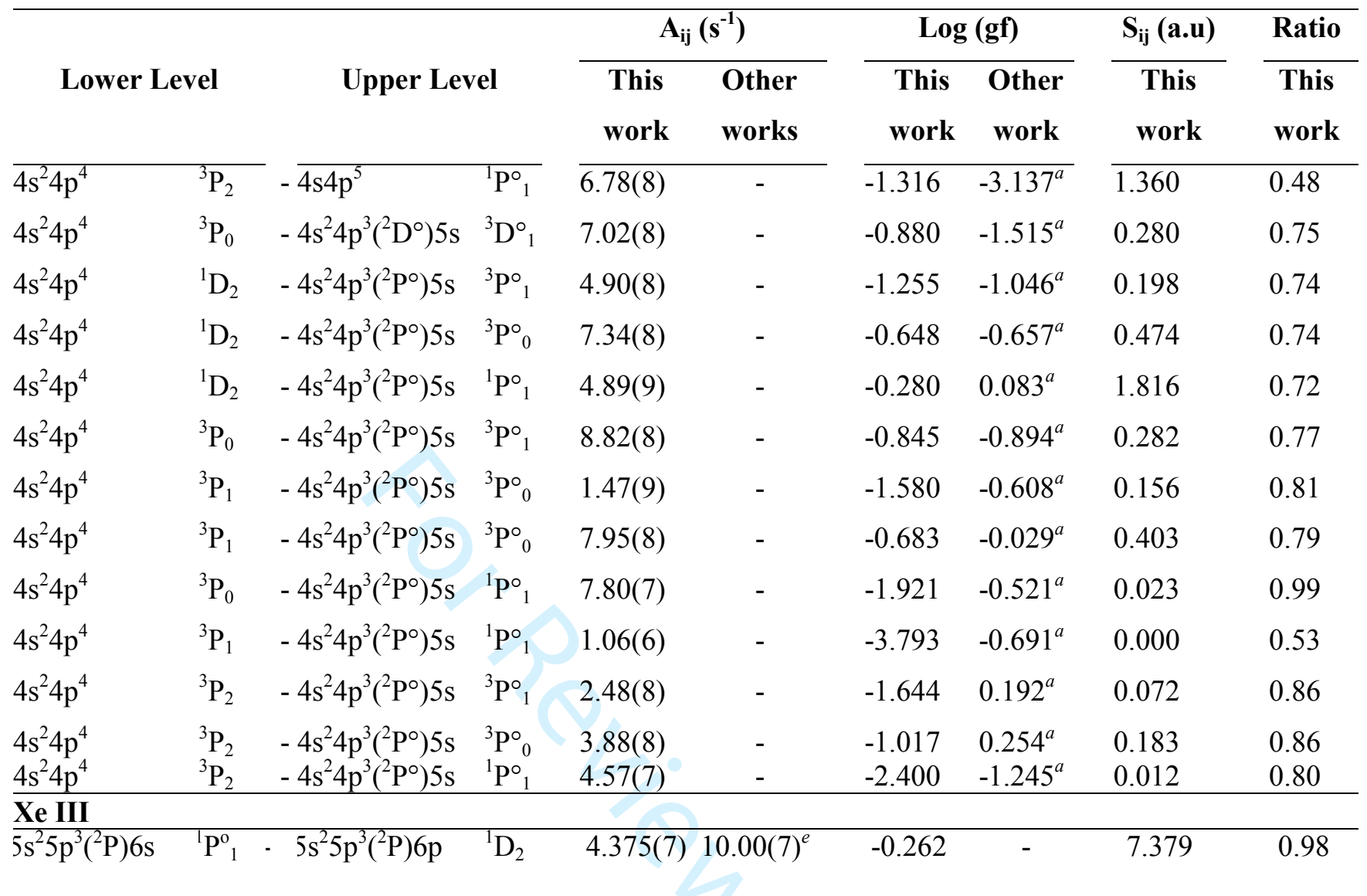

${ }^{a}$ [17], ${ }^{b}[16],{ }^{c}[12],{ }^{d}[2],{ }^{e}[24]$ 